\title{
Stereocontrolled Assembly of Tetrasubstituted Tetrahydrofurans: A Concise Synthesis of Virgatusin
}

Tito Akindele, ${ }^{\dagger}$ Stephen P. Marsden ${ }^{\star, \dagger}$ and John G. Cumming ${ }^{\ddagger}$

School of Chemistry, University of Leeds, Leeds LS2 9JT, U.K., and

AstraZeneca, Mereside, Alderley Park, Macclesfield SK10 4TG, U.K.

s.p.marsden@leeds.ac.uk

Supporting information and spectra 
General methods for tetrahydrofuran synthesis: $\left(4 S, 2^{\prime} R, 3^{\prime} S, 4^{\prime} S, 5^{\prime} S\right)$-4-benzyl-3(2',5'-diphenethyl-4' -vinyltetrahydrofuran-3'-carbonyl)-oxazolidin-2-one 11a

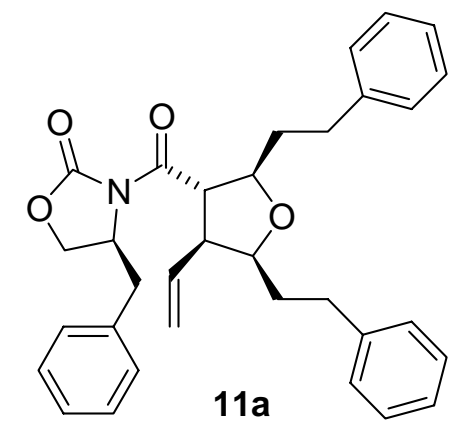

\section{Method 1 - Mediated by boron trifluoride etherate (Table 1, entry 1)}

Boron trifluoride etherate $(1.90 \mathrm{ml}, 14.1 \mathrm{mmol})$ and hydrocinnamaldehyde $(1.85 \mathrm{ml}, 14.0$ mmol) were sequentially added to a solution of 9 (3.15 g, $7.01 \mathrm{mmol})$ in dry dichloromethane $(150 \mathrm{ml})$ at $-78{ }^{\circ} \mathrm{C}$. The reaction mixture was stirred for 2 hours at -78 ${ }^{\circ} \mathrm{C}$ and for $13^{1} \frac{1}{2}$ hours at room temperature. The reaction mixture was quenched with brine $(100 \mathrm{ml})$ and then extracted with ethyl acetate $(3 \times 50 \mathrm{ml})$. The combined organic extracts were washed with water $(50 \mathrm{ml})$, then dried $\left(\mathrm{MgSO}_{4}\right)$, filtered and evaporated under reduced pressure to give the crude product which was purified by flash chromatography eluting with 20:80 diethyl ether-petrol, to give 11a (3.26 g, $91 \%)$ as a pale yellow oil, $R_{\mathrm{F}} 0.41$ (30\% ethyl acetate in hexane); $[\alpha]^{18}{ }_{\mathrm{D}}+33\left(c 3.4, \mathrm{CHCl}_{3}\right) ; v_{\max } / \mathrm{cm}^{-}$ ${ }^{1}$ (neat) $3082 \mathrm{~s}, 3062 \mathrm{~s}, 2927 \mathrm{~s}, 2861 \mathrm{w}, 1736 \mathrm{~s}, 1693 \mathrm{~s}, 1639 \mathrm{~m}, 1603 \mathrm{~s}, 1584 \mathrm{w}, 1495 \mathrm{~s}$, $1454 \mathrm{~s}, 1385 \mathrm{~s}, 1347 \mathrm{~s}, 918 \mathrm{~s}, 748 \mathrm{~s}$, and $699 \mathrm{~s} ; \delta_{\mathrm{H}}\left(500 \mathrm{MHz}, \mathrm{CDCl}_{3}\right)$ 7.25-7.07 $(15 \mathrm{H}$, m), $5.80(1 \mathrm{H}, \mathrm{dt}, J 16.5,10.5), 5.033(1 \mathrm{H}, \mathrm{d}, J 10.5), 5.028(1 \mathrm{H}, \mathrm{d}, J 16.5), 4.55(1 \mathrm{H}$, m), $4.24(1 \mathrm{H}, \mathrm{t}, J$ 7.5), $4.14(1 \mathrm{H}, \mathrm{td}, J$ 7.5, 5.0), 4.07-4.02 (3 H, m), $3.14(1 \mathrm{H}, \mathrm{dd}, J$ 13.5, 3.0), 3.09 (1 H, dt, $J$ 10.5, 7.5), 2.81-2.73 (2 H, m), 2.63-2.56 (3 H, m), 1.97-1.83 (2 $\mathrm{H}, \mathrm{m}), 1.77(1 \mathrm{H}, \mathrm{m})$ and $1.66(1 \mathrm{H}, \mathrm{m},) ; \delta_{\mathrm{C}}\left(75 \mathrm{MHz}, \mathrm{CDCl}_{3}\right) 174.1,153.8,142.5,142.2$, 136.2, 135.5, 129.9, 129.4, 129.0, 128.9, 128.8, 127.9, 127.8, 126.3, 126.28, 117.9, 82.2, 81.3, 66.2, 55.9, 55.0, 53.0, 38.4, 37.5, 34.4, 33.0 and 32.9; m/z (ES+) 532 (73\%, $\left.[\mathrm{M}+\mathrm{Na}]^{+}\right), 527\left(58,\left[\mathrm{M}+\mathrm{NH}_{4}\right]^{+}\right), 510\left(100,[\mathrm{M}+\mathrm{H}]^{+}\right), 376(91)$ and $309(33)$; HRMS (ES+) found $[\mathrm{M}+\mathrm{Na}]^{+}$532.2441, $\mathrm{C}_{33} \mathrm{H}_{35} \mathrm{NO}_{4} \mathrm{Na}$ requires 532.2464. Anal. Calcd for $\mathrm{C}_{33} \mathrm{H}_{35} \mathrm{NO}_{4}$ : C, 77.8; H, 6.9; N, 2.8. Found: C, 77.5; H, 7.1; N, 2.7. 


\section{Method 2 - Mediated by trimethylsilyl triflate (Table 2, entry 1)}

Trimethylsilyl trifluoromethanesulfonate $(5 \mu \mathrm{l}, 0.03 \mathrm{mmol}, 20 \mathrm{~mol} \%)$ was added to a solution of (4S, 6'S, 7'R)-4-benzyl-3-(2',2'-dimethyl-7'-phenethyl-2',3',6',7'-tetrahydro[1',2']oxasilepine-6'-carbonyl)oxazolidin-2-one $\quad 9 \quad(0.062 \quad \mathrm{~g}, \quad 0.14 \quad \mathrm{mmol})$ and hydrocinnamaldehyde $(18 \mu \mathrm{l}, 0.14 \mathrm{mmol})$ in dry dichloromethane $(4 \mathrm{ml})$ at $-78{ }^{\circ} \mathrm{C}$. The reaction mixture was stirred for $14 \frac{1}{4}^{1}$ hours at $-78{ }^{\circ} \mathrm{C}$. The reaction mixture was quenched with brine $(10 \mathrm{ml})$ and then extracted with ethyl acetate $(3 \times 10 \mathrm{ml})$. The combined organic extracts were washed with water $(10 \mathrm{ml})$, then dried $\left(\mathrm{MgSO}_{4}\right)$, filtered and evaporated under reduced pressure to give the crude product which was purified by flash column chromatography eluting with gradient elution: 20:80 to 35:75 diethyl etherpetrol to give 11a $(0.060 \mathrm{~g}, 86 \%)$ as a pale yellow oil whose data agreed with that reported above.

(4S, 2'R, 3'S, 4'S, 5'R)-4-benzyl-3-(5'-benzyloxymethyl-2'-phenethyl-4'vinyltetrahydrofuran-3' -carbonyl)-oxazolidin-2-one 12a

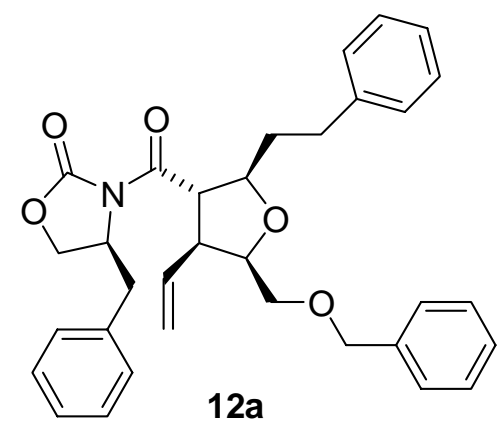

\section{Method 1 (Table 1, entry 2)}

Carried out according to the general method using boron trifluoride etherate $(9 \mu 1,0.07$ mmol), benzyloxyacetaldehyde $(0.0065 \mathrm{~g}, 0.043 \mathrm{mmol}), 9(0.015 \mathrm{~g}, 0.033 \mathrm{mmol})$ in dry dichloromethane $(1.5 \mathrm{ml})$ at $-78{ }^{\circ} \mathrm{C}$. The reaction mixture was stirred for $2 \frac{1}{2}$ hours at $-78{ }^{\circ} \mathrm{C}$ and for $16 \frac{1}{4}$ hours at room temperature. After work up the crude product was purified by flash chromatography eluting 30:70 diethyl ether-petrol, to give 12a $(0.011 \mathrm{~g}$, $65 \%)$ as a pale yellow oil viscous oil, $R_{\mathrm{F}} 0.29$ (50\% diethyl ether in petrol); $[\alpha]^{18}{ }_{\mathrm{D}}+33(c$ 0.45, $\mathrm{CH}_{2} \mathrm{Cl}_{2}$ ); $v_{\max } / \mathrm{cm}^{-1}$ (neat) $3028 \mathrm{~m}, 2925 \mathrm{~m}, 2862 \mathrm{~m}, 1782 \mathrm{~s}, 1721 \mathrm{~m}, 1692 \mathrm{~s}, 1639$ w, 1603 w, 1496 m, 1454 m, 1385 s, 1351 s, 1290 m, 1270 m, 1210 s, 1104 s, 1053 m, 
$911 \mathrm{~s}, 733 \mathrm{~s}$, and $700 \mathrm{~s} ; \delta_{\mathrm{H}}\left(500 \mathrm{MHz}, \mathrm{CDCl}_{3}\right) ; 7.32-7.07(15 \mathrm{H}, \mathrm{m}), 5.84(1 \mathrm{H}, \mathrm{dt}, J$ 17.5, 10.0), 5.06 (1 H, d, J 17.0), 5.02 (1 H, d, J 10.0), 4.57 (1 H, m), 4.53-4.48 (2 H, m), 4.36 (1 H, t, J 8.0), 4.27 (1 H, dt, J 9.0, 4.5), 4.21 (1 H, td, $J$ 8.0, 4.5), 4.09-4.03 (2 H, m), 3.47 (1 H, d, J 7.0), 3.46 (1 H, d, J 7.0), 3.23 (1 H, m), 3.17 (1 H, dd, J 13.0, 3.0), 2.76 (1 $\mathrm{H}, \mathrm{m}), 2.60(1 \mathrm{H}, \mathrm{dd}, J 13.0,10.0), 2.56(1 \mathrm{H}, \mathrm{m})$ and 1.99-1.82 $(2 \mathrm{H}, \mathrm{m}) ; \delta_{\mathrm{C}}(75 \mathrm{MHz}$, $\left.\mathrm{CDCl}_{3}\right) 173.8,153.8,142.2,138.6,135.52,135.47,129.8,129.4,128.9,128.8,128.7$, 128.1, 127.9, 127.8, 126.2, 118.4, 82.9, 80.9, 73.8 C, 71.0 C, 66.2, 55.9, 54.4, 52.6, 38.4, 36.8, and 33.0; m/z (ES+) $548\left(58 \%,[\mathrm{M}+\mathrm{Na}]^{+}\right), 544\left(32,\left[\mathrm{M}+\mathrm{NH}_{4}\right]^{+}\right), 527\left(41,[\mathrm{M}+\mathrm{H}]^{+}\right)$, $526\left(100,[\mathrm{M}+\mathrm{H}]^{+}\right), 508\left(33,[\mathrm{M}-\mathrm{OH}]^{+}\right), 466(52), 349\left(88,\left[\mathrm{M}-\mathrm{PhCH}_{2} \mathrm{CHCH}_{2} \mathrm{CO}_{2} \mathrm{~N}\right]^{+}\right)$, 332 (56) and 302 (33); HRMS (ES+) found $[\mathrm{M}+\mathrm{H}]^{+} 526.2609, \mathrm{C}_{33} \mathrm{H}_{36} \mathrm{NO}_{4}$ requires 526.2593.

\section{Method 2 (Table 2, entry 2)}

Carried out on according to the general method using TMSOTf $(50 \mu 1,0.28 \mathrm{mmol}, 5$ mol\%), 9 (2.65 g, $5.89 \mathrm{mmol})$ and benzyloxyacetaldehyde $(0.889 \mathrm{~g}, 5.92 \mathrm{mmol})$ in dry dichoromethane $(80 \mathrm{ml})$ at $-78{ }^{\circ} \mathrm{C}$. The reaction mixture was stirred for $2 \frac{3}{4}$ hours at -78 ${ }^{\circ} \mathrm{C}$. A further aliquot of TMSOTf $(150 \mu \mathrm{l}, 0.827 \mathrm{mmol}, 14 \mathrm{~mol} \%)$ was added to the reaction mixture which was stirred for a further $2 \frac{1}{4}$ hours at $-78{ }^{\circ} \mathrm{C}$. Further TMSOTf (200 $\mu 1,1.10 \mathrm{mmol}, 19 \mathrm{~mol} \%)$ was added to the reaction mixture which was stirred for a further 4 hours at $-78{ }^{\circ} \mathrm{C}$ and for a further $8 \frac{1}{4}$ hours at room temperature. Work-up and purification by flash column chromatography eluting with 30:70 diethyl ether-petrol gave 12a $(2.35 \mathrm{~g}, 76 \%)$ as a pale yellow viscous oil whose data agreed with that reported above. 
$\left(4 S, 2^{\prime} R, 3^{\prime} S, 4^{\prime} S, 5^{\prime} R\right)$ and $\left(4 S, 2^{\prime} R, 3^{\prime} S, 4^{\prime} S, 5^{\prime} S\right)$-4-benzyl-3-(2'-phenethyl-5'-phenyl4 '-vinyltetra-hydrofuran-3'-carbonyl)-oxazolidin-2-one 13a and 13b
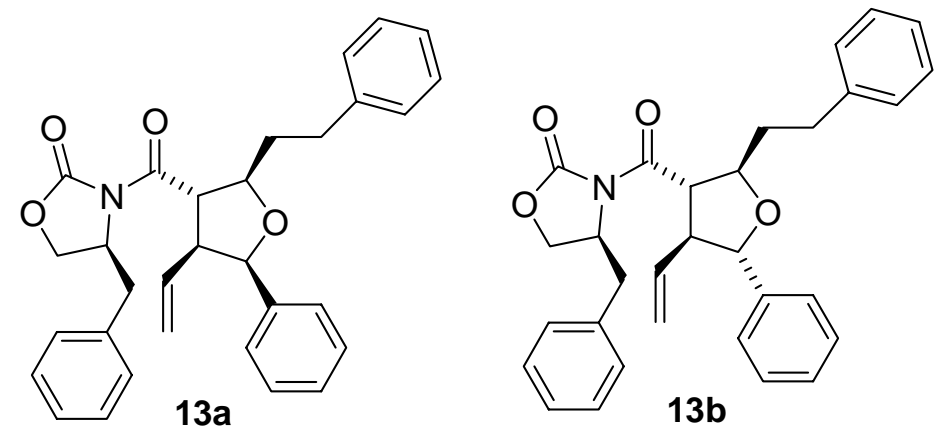

\section{Method 1 (Table 1, entry 3)}

Carried out according to the general method using boron trifluoride etherate $(0.360 \mathrm{ml}$, $2.67 \mathrm{mmol})$, benzaldehyde $(0.270 \mathrm{ml}, 2.66 \mathrm{mmol})$ and $9(0.034 \mathrm{~g}, 0.076 \mathrm{mmol})$ in dry dichloromethane $(1 \mathrm{ml})$ at $-78{ }^{\circ} \mathrm{C}$. The reaction mixture was stirred for $4 \frac{1}{2}$ hours at -78 ${ }^{\circ} \mathrm{C}$ and at room temperature for $22 \frac{1}{2}$ hours. Work-up and flash chromatography eluting with 15:85 ethyl acetate-petrol gave 13a and 13b $(0.024 \mathrm{~g}, 65 \%$ as a 93:7 mixture of diastereoisomers), as a pale yellow viscous oil, $R_{\mathrm{F}} 0.07$ (10\% ethyl acetate in hexane); $[\alpha]^{21}{ }_{\mathrm{D}}+45\left(c 0.65, \mathrm{CH}_{2} \mathrm{Cl}_{2}\right) ; v_{\max } / \mathrm{cm}^{-1}$ (neat) $3063 \mathrm{~m}, 3028 \mathrm{~m}, 2923 \mathrm{~m}, 1779 \mathrm{~s}, 1694 \mathrm{~s}$, 1640 w, 1603 w, 1495 s, 1454 s, 1385 s, 1351 s, 1209 s, 1106 s and 700 s; $\delta_{\mathrm{H}}(500 \mathrm{MHz}$, $\mathrm{CDCl}_{3}$, signals for 13a) 7.29-7.09 $(15 \mathrm{H}, \mathrm{m}), 5.23(1 \mathrm{H}, \mathrm{d}, J$ 8.0), $5.21(1 \mathrm{H}, \mathrm{dt}, J$ 17.0, 10.0), 4.90 (1 H, dd, $J$ 17.0, 1.5), 4.79 (1 H, dd, $J 10.0,1.5), 4.60(1 \mathrm{H}, \mathrm{m}), 4.40(1 \mathrm{H}, \mathrm{t}, J$ 8.0), 4.29 (1 H, td, $J$ 8.0, 4.5), 4.08 (1 H, t, $J 9.0), 4.04(1 \mathrm{H}, \mathrm{dd}, J$ 9.0, 3.0), $3.44(1 \mathrm{H}$, m), $3.18(1 \mathrm{H}, \mathrm{dd}, J 13.5,3.5), 2.87$ (1H, ddd, $J$ 14.0, 10.5, 4.5), 2.67 (1 H, ddd, $J$ 14.0, 10.5, 6.5), $2.59(1 \mathrm{H}, \mathrm{dd}, J$ 13.5, 9.5), $2.11(1 \mathrm{H}, \mathrm{m}), 1.99(1 \mathrm{H}, \mathrm{m})$; signals for 13b visible at $5.84(1 \mathrm{H}, \mathrm{dt}, J 17.0,10.0), 5.01(1 \mathrm{H}, \mathrm{d}, J 10.0)$ and $2.54(1 \mathrm{H}, \mathrm{dd}, J 13.5,9.5) ; \delta_{\mathrm{C}}(75$ $\left.\mathrm{MHz}, \mathrm{CDCl}_{3}\right) ; 173.2,153.0,141.7,139.2,136.1,135.0,129.4,129.0,128.5,128.4$, $128.1,127.8,127.4,126.8,125.9,117.0,83.3,82.2,65.8,56.2,55.8,52.2,38.0,36.2$ and $32.5 ; \mathrm{m} / \mathrm{z}(\mathrm{ES}+) 504\left(100 \%,[\mathrm{M}+\mathrm{Na}]^{+}\right)$; HRMS (ES+) found $[\mathrm{M}+\mathrm{Na}]^{+}$504.2136, $\mathrm{C}_{31} \mathrm{H}_{31} \mathrm{NO}_{4} \mathrm{Na}$ requires 504.2151 . 


\section{Method 1 - extended reaction time (Table 1, entry 4)}

Carried out according to the general method using boron trifluoride etherate $(0.360 \mathrm{ml}$, $2.67 \mathrm{mmol})$, benzaldehyde $(0.270 \mathrm{ml}, 2.66 \mathrm{mmol})$ and $9(1.20 \mathrm{~g}, 2.67 \mathrm{mmol})$ in dry dichloromethane $(60 \mathrm{ml})$ at $-78{ }^{\circ} \mathrm{C}$. The reaction mixture was stirred for $271 / 4$ hours at $-78{ }^{\circ} \mathrm{C}$ and at room temperature for $901 / 4$ hours. The reaction mixture was quenched with brine $(30 \mathrm{ml})$ and then extracted with ethyl acetate $(3 \times 30 \mathrm{ml})$. The combined organic extracts were washed with water $(30 \mathrm{ml})$, dried $\left(\mathrm{MgSO}_{4}\right)$, filtered and evaporated under reduced pressure to give the crude product which was purified by flash chromatography eluting with 15:85 ethyl acetate-petrol, to give 13a, 13b and an unidentified diastereoisomer $13 \mathrm{c}(1.00 \mathrm{~g}, 78 \%$ as an 80:12:8 mixture of diastereoisomers) as a pale yellow viscous oil, $R_{\mathrm{F}} 0.10$ (10\% ethyl acetate in hexane); $[\alpha]^{21}{ }_{\mathrm{D}}+41\left(c 6.2, \mathrm{CH}_{2} \mathrm{Cl}_{2}\right)$; signals for $13 \mathrm{c}$ visible at $\delta_{\mathrm{H}}\left(500 \mathrm{MHz}, \mathrm{CDCl}_{3}\right) 5.72(1 \mathrm{H}, \mathrm{dt}, J 17.0,10.0), 5.03(1 \mathrm{H}, \mathrm{d}, J$ 10.0), $3.33(1 \mathrm{H}, \mathrm{dd}, J 13.5,3.5)$ and $2.40(1 \mathrm{H}, \mathrm{dd}, J 13.5,11.0)$, whose other data agree with that reported above.

\section{Method 1 - reaction at reflux (Table 1, entry 5)}

Carried out according to the general method using boron trifluoride etherate $(5 \mu \mathrm{l}, 0.04$ mmol), benzaldehyde $(4 \mu \mathrm{l}, 0.04 \mathrm{mmol})$ and $9(0.017 \mathrm{~g}, 0.038 \mathrm{mmol})$ in dry dichloromethane $(2 \mathrm{ml})$ at room temperature. The reaction mixture was stirred for $1 \frac{1}{2}$ hours at room temperature and then refluxed for $165^{1} / 4$ hours. Work-up and flash chromatography eluting with 15:85 ethyl acetate-petrol, gave 13a, 13b and 13c $(0.010 \mathrm{~g}$, $74 \%$ as a 73:15:12 mixture of diastereoisomers), as a pale yellow viscous oil, $R_{\mathrm{F}} 0.15$ (30\% diethyl ether in petrol); $[\alpha]^{21}+43\left(c 0.15, \mathrm{CH}_{2} \mathrm{Cl}_{2}\right)$, whose other data agree with that reported above.

\section{Method 2 (Table 2, entry 3)}

Carried out according to the general method using trimethylsilyl trifluoromethanesulfonate $(5 \mu \mathrm{l}, 0.03 \mathrm{mmol}, 8 \mathrm{~mol} \%), 9(0.15 \mathrm{~g}, 0.34 \mathrm{mmol})$ and benzaldehyde $(35 \mu 1,0.34 \mathrm{mmol})$ in dry dichloromethane $(8.0 \mathrm{ml})$ at $-78^{\circ} \mathrm{C}$. The reaction mixture was stirred for $5 \frac{1}{2}$ hours at $-78{ }^{\circ} \mathrm{C}$. Work-up and flash column chromatography 
eluting with 20:80 diethyl ether-petrol gave 13a $(0.13 \mathrm{~g}, 81 \%)$ as a pale yellow viscous oil; $[\alpha]^{19}{ }_{\mathrm{D}}+23\left(c 0.22, \mathrm{CH}_{2} \mathrm{Cl}_{2}\right)$, whose other data agreed with that reported above.

$\left(4 S, 2^{\prime} R, 3^{\prime} S, 4^{\prime} S, 5^{\prime} R\right)$ and $\left(4 S, 2^{\prime} R, 3^{\prime} S, 4^{\prime} S, 5^{\prime} S\right)$-3-(5'-benzo-[1,3]-dioxol-5-yl-2'phenethyl-4'-vinyltetrahydrofuran-3'-carbonyl)-4-benzyloxazolidin-2-one 14a and 14b
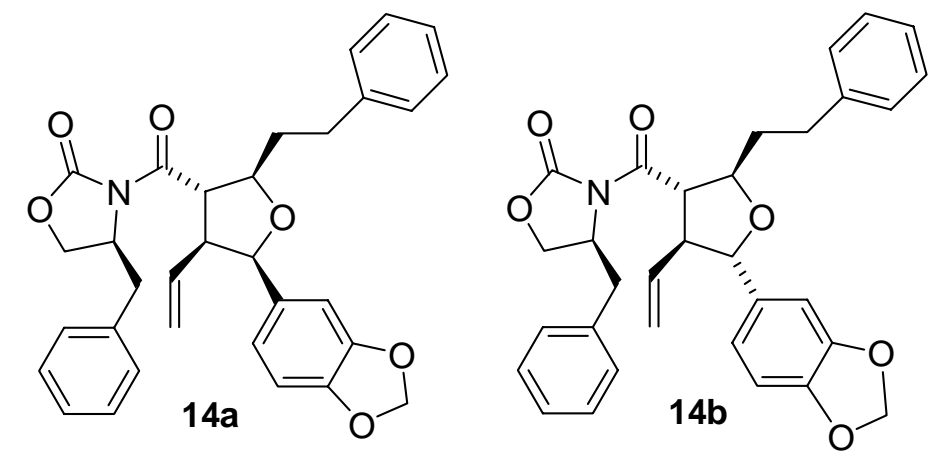

\section{Method 1 (Table 1, entry 6)}

Carried out according to the general method using boron trifluoride etherate ( $31 \mu 1,0.23$ $\mathrm{mmol})$, piperonal $(0.035 \mathrm{~g}, 0.23 \mathrm{mmol}), 9(0.10 \mathrm{~g}, 0.23 \mathrm{mmol})$ in dry dichloromethane $(7$ ml) at $-78{ }^{\circ} \mathrm{C}$. The reaction mixture was stirred for 3 hours at $-78{ }^{\circ} \mathrm{C}$ and for $124{ }^{1} / 4$ hours at room temperature. Work-up and flash chromatography eluting with gradient elution: 20:80 to 50:50 ethyl acetate-petrol gave 14a and $\mathbf{1 4 b}\left(0.051 \mathrm{~g}, 37 \%\right.$ by ${ }^{1} \mathrm{H} \mathrm{nmr}$, as a 9:91 diastereomeric mixture) and an unidentified product, as a pale yellow oil, $R_{\mathrm{F}} 0.41(30 \%$ ethyl acetate in hexane); $[\alpha]^{19}{ }_{\mathrm{D}}+73\left(c 2.2, \mathrm{CH}_{2} \mathrm{Cl}_{2}\right) ; v_{\max } / \mathrm{cm}^{-1}$ (neat) $3027 \mathrm{w}, 2925 \mathrm{~m}$, 1779 s, 1694 s, 1642 w, 1603 w, 1490 s, 1444 s, 1385 s, 1250 s, 1039 s and 700 s; $\delta_{\mathrm{H}}(500$ $\mathrm{MHz}, \mathrm{CDCl}_{3}$, signals for 14a); 7.35-7.16 $(10 \mathrm{H}, \mathrm{m}), 6.98-6.76(3 \mathrm{H}, \mathrm{m}), 5.95(2 \mathrm{H}, \mathrm{s})$, $5.87(1 \mathrm{H}, \mathrm{dt}, J 17.0,9.5), 5.08(1 \mathrm{H}, \mathrm{d}, J 10.0), 4.98(1 \mathrm{H}, \mathrm{d}, J 17.0), 4.75(1 \mathrm{H}, \mathrm{d}, J 9.5)$, 4.69-4.55 (2 H, m), 4.62 (1 H, t, J 9.0), 4.09-4.17 (2 H, m), $3.23(1 \mathrm{H}, \mathrm{dd}, J$ 13.5, 3.5), $3.17(1 \mathrm{H}, \mathrm{q}, J$ 9.5), $2.87(1 \mathrm{H}, \mathrm{m}), 2.69(1 \mathrm{H}, \mathrm{m}), 2.61(1 \mathrm{H}, \mathrm{dd}, J$ 13.5, 9.5), $2.05(1 \mathrm{H}$, $\mathrm{m}), 1.96(1 \mathrm{H}, \mathrm{m})$; signals for $\mathbf{1 4 b}$ visible at $5.33(1 \mathrm{H}, \mathrm{dt}, J 17.0,10.0), 5.22(1 \mathrm{H}, \mathrm{d}, J$ 8.0), $4.90(1 \mathrm{H}, \mathrm{d}, J 10.0)$ and $4.44(1 \mathrm{H}, \mathrm{t}, J 8.5) ;\left(75 \mathrm{MHz}, \mathrm{CDCl}_{3}\right)$ 173.0, 153.4, 148.0, 147.7, 135.5, 135.3, 134.7, 129.8, 129.4, 128.9, 128.8, 128.6, 127.8, 126.3, 120.5, 119.0, $108.4,107.2,101.4,85.6,82.7,66.3,61.1,56.0,54.0,38.5,37.3$, and $32.3 ; \mathrm{m} / \mathrm{z}(\mathrm{ES}+)$ 602 (56), 404 (36, [M- $\left.\left.\mathrm{C}_{6} \mathrm{H}_{3} \mathrm{CH}_{2}(\mathrm{O})_{2}\right]^{+}\right), 391$ (100), 159 (51), 114 (39) and 101 (61). 


\section{Method 2 (Table 2, entry 4)}

Carried out according to the general method using trimethylsilyl trifluoromethanesulfonate $(5 \mu \mathrm{l}, 0.03 \mathrm{mmol}, 15 \mathrm{~mol} \%), 9(0.080 \mathrm{~g}, 0.18 \mathrm{mmol})$ and piperonal $(0.027 \mathrm{~g}, 0.18 \mathrm{mmol})$ in dry dichloromethane $(5 \mathrm{ml})$ at $-78{ }^{\circ} \mathrm{C}$. The reaction mixture was stirred for $14 \frac{1}{4}$ hours at $-78{ }^{\circ} \mathrm{C}$. Further trimethylsilyl trifluoromethanesulfonate ( $5 \mu \mathrm{l}, 27.6 \mu \mathrm{mol}, 15 \mathrm{~mol} \%)$ was added to the reaction mixture. The reaction mixture was stirred for $7 \frac{3}{4}$ hours at $-78{ }^{\circ} \mathrm{C}$. Work-up and flash column chromatography eluting with gradient elution: 20:80 to 50:50 diethyl ether-petrol, gave 14a $(0.068 \mathrm{~g}, 72 \%)$ as colourless viscous oil, $R_{\mathrm{F}} 0.30$ (50\% diethyl ether in petrol); $[\alpha]^{20}{ }_{589}+16\left(c 0.15, \mathrm{CH}_{2} \mathrm{Cl}_{2}\right) ; v_{\max } / \mathrm{cm}^{-1}$ (neat) $3071 \mathrm{w}, 2919 \mathrm{~m}, 1779 \mathrm{~s}, 1691 \mathrm{~s}, 1637 \mathrm{w}$, 1603 w, 1503 m, 1490 s, 1444 s, 1385 s, 1239 s, 1038 s and 700 s; $\delta_{\mathrm{H}}\left(500 \mathrm{MHz}, \mathrm{CDCl}_{3}\right)$; 7.25-7.09 (10 H, m), $6.73(1 \mathrm{H}, \mathrm{s}), 6.70(1 \mathrm{H}, \mathrm{d}, J 7.5), 6.67(1 \mathrm{H}, \mathrm{d}, J 7.5), 5.87(2 \mathrm{H}, \mathrm{s})$, $5.26(1 \mathrm{H}, \mathrm{m}), 5.14(1 \mathrm{H}, \mathrm{d}, J$ 8.0), $4.91(1 \mathrm{H}, \mathrm{d}, J 16.5), 4.82(1 \mathrm{H}, \mathrm{d}, J 10.0), 4.59(1 \mathrm{H}$, m), 4.37(1 H, t, J 8.5), $4.25(1 \mathrm{H}, \mathrm{m}), 4.09-4.03(2 \mathrm{H}, \mathrm{m}), 3.38(1 \mathrm{H}, \mathrm{q}, J$ 8.5), $3.17(1 \mathrm{H}$, dd, $J$ 13.5, 3.5), 2.86 (1 H, ddd, 14.5, 9.5, 5.0), $2.65(1 \mathrm{H}, \mathrm{m}), 2.58(1 \mathrm{H}, \mathrm{dd}, J 13.5,10.0)$, $2.08(1 \mathrm{H}, \mathrm{m})$ and $1.96(1 \mathrm{H}, \mathrm{m}) ; \delta_{\mathrm{C}}\left(75 \mathrm{MHz}, \mathrm{CDCl}_{3}\right) 173.6,153.4,148.0,147.2,142.1$, 136.6, 135.5, 133.7, 129.8, 129.4, 128.9, 128.8, 127.9, 126.3, 120.5, 117.4, 108.4, 107.8, $101.4,83.6,82.5,66.3,56.7,55.4,52.5,38.4,36.6$ and 33.0; $\mathrm{m} / \mathrm{z}(\mathrm{ES}+) 548(60 \%$, $\left.[\mathrm{M}+\mathrm{Na}]^{+}\right), \quad 508 \quad\left(35, \quad[\mathrm{M}-\mathrm{OH}]^{+}\right), \quad 405 \quad\left(32, \quad\left[\mathrm{M}-\mathrm{C}_{6} \mathrm{H}_{3} \mathrm{CH}_{2} \mathrm{O}_{2}\right]^{+}\right), \quad 404 \quad(100$, $\left[\mathrm{M}-\mathrm{C}_{6} \mathrm{H}_{3} \mathrm{CH}_{2} \mathrm{O}_{2}\right]^{+}$), 376 (43) and 331 (84); HRMS (ES+) found [M+Na] $]^{+}$548.2067, $\mathrm{C}_{32} \mathrm{H}_{31} \mathrm{NO}_{6} \mathrm{Na}$ requires 548.2049.

$\left(4 S, 2^{\prime} R, 3^{\prime} S, 4^{\prime} S, 5^{\prime} S\right)$ and $\left(4 S, 2^{\prime} R, 3^{\prime} S, 4 ' S, 5^{\prime} R\right)$-4-benzyl-3-(2'-phenethyl-5'-styryl4 '-vinyltetrahydrofuran-3' -carbonyl)-oxazolidin-2-one $15 \mathrm{a}$ and $15 \mathrm{~b}$
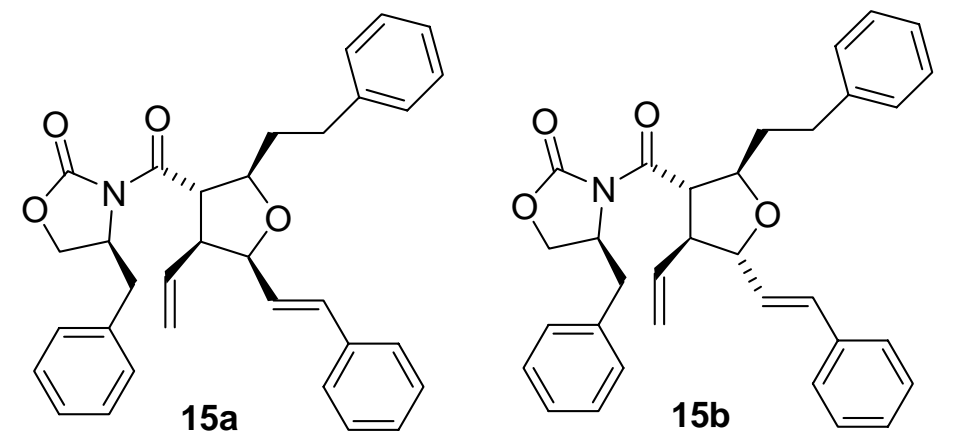


\section{Method 1 (Table 1, entry 7)}

Carried out according to the general method using boron trifluoride etherate $(17 \mu 1,0.13$ mmol), trans-cinnamaldehyde $(16 \mu 1,0.13 \mathrm{mmol})$ and $9(0.056 \mathrm{~g}, 0.13 \mathrm{mmol})$ in dry dichloromethane $(3.5 \mathrm{ml})$ at $-78{ }^{\circ} \mathrm{C}$. The reaction mixture was stirred for 1 hour at $-78{ }^{\circ} \mathrm{C}$ and for $187^{1} / 2$ hours at room temperature. Work-up and flash chromatography eluting with gradient elution: 15:85 to 30:70 diethyl ether-petrol, gave 15a, 15b and an unidentified diastereoisomer 15c $(0.025 \mathrm{~g}, 39 \%$ as an 11:78:11 mixture of diastereoisomers), as a pale yellow oil, $R_{\mathrm{F}} 0.16$ (30\% diethyl ether in petrol); $[\alpha]^{24}+71(c$ 1.7, $\mathrm{CH}_{2} \mathrm{Cl}_{2}$ ); $v_{\max } / \mathrm{cm}^{-1}$ (neat) $3027 \mathrm{~m}, 2924 \mathrm{~m}, 1779 \mathrm{~s}, 1693 \mathrm{~s}, 1641 \mathrm{w}, 1495 \mathrm{~m}, 1454 \mathrm{~m}$, 1386 s, 1350 m, 1209 m, 1107 m, 749 m and 699 s; $\delta_{\mathrm{H}}\left(500 \mathrm{MHz}, \mathrm{CDCl}_{3}\right.$, signals for 15b) 7.34-7.08 (15 H, m), 6.54 (1 H, d, J 16.0), 6.17 (1 H, dd, $J 16.0,7.5), 5.79$ (1 H, dt, $J$ 17.0, 10.0), 5.07 (1 H, d, J 17.0), 5.06 (1 H, d, J 10.0), 4.60 (1 H, m), 4.50 (1 H dd, $J$ 10.0, 8.5), 4.41-4.36 (2 H, m), 4.10-4.03 (2 H, m), 3.18 (1 H, dd, J 13.5, 3.5), 3.06 (1 H, q, $J$ 10.0), 2.79 (1 H, m), 2.59 (1 H, m), 2.57 (1 H, dd, 13.5, 9.5), $1.95(1 \mathrm{H}, \mathrm{m})$ and 1.86 $(1 \mathrm{H}, \mathrm{m})$; signals for 15a visible at $6.56(1 \mathrm{H}, \mathrm{d}, J 16.0), 6.12(1 \mathrm{H}, \mathrm{dd}, J 16.0,7.5), 4.74$ $(1 \mathrm{H}, \mathrm{t}, J$ 7.5); signals for $15 \mathrm{c}$ visible at $5.65(1 \mathrm{H}, \mathrm{dt}, J 17.0,10.0), 4.26(1 \mathrm{H}, \mathrm{m}), 3.40(1$ $\mathrm{H}, \mathrm{m}), 3.31(1 \mathrm{H}, \mathrm{dd}, J$ 13.0, 3.0), $2.86(1 \mathrm{H}, \mathrm{m}), 2.65(1 \mathrm{H}, \mathrm{m})$ and $2.40(1 \mathrm{H}, \mathrm{dd}, J$ 13.0, $10.5) ; \delta_{\mathrm{C}}\left(75 \mathrm{MHz}, \mathrm{CDCl}_{3}\right) 173.0,153.5,142.1,136.9,135.5,135.3,132.9,129.8,129.4$, 128.94, 128.88, 128.80, 128.2, 128.1, 127.9, 127.1, 126.3, 119.0, 84.9, 82.7, 66.3, 59.0, 56.0, 53.7, 38.5, 37.2 and 32.7; m/z (ES+) $508\left(55 \%,[\mathrm{M}+\mathrm{H}]^{+}\right), 405$ (32), 404 (100) and 374 (44); HRMS (ES+) found $[\mathrm{M}+\mathrm{Na}]^{+} 508.2508, \mathrm{C}_{33} \mathrm{H}_{33} \mathrm{NO}_{4}$ requires 508.2488.

\section{Method 2 (Table 2, entry 5)}

Carried out according to the general method using trimethylsilyl trifluoromethanesulfonate $(5 \mu \mathrm{l}, 0.03 \mathrm{mmol}, 6 \mathrm{~mol} \%), 9(0.20 \mathrm{~g}, 0.45 \mathrm{mmol})$ and transcinnamaldehyde $(65 \mu \mathrm{l}, 0.52 \mathrm{mmol})$ in dry dichloromethane $(7 \mathrm{ml})$ at $-78{ }^{\circ} \mathrm{C}$. The reaction mixture was stirred for 6 hours at $-78{ }^{\circ} \mathrm{C}$. Work-up and flash column chromatography eluting with gradient elution: 20:80 to 50:50 diethyl ether-petrol, gave (4S, 2'R, 3'S, 4'S, 5'S)-4-benzyl-3-(2'-phenethyl-5'-styryl-4'-vinyltetrahydrofuran-3'carbonyl)-oxazolidin-2-one $\mathbf{1 5 a}$ and $\mathbf{1 5 b}(0.22 \mathrm{~g}, 95 \%$ as a $97: 3$ mixture of diastereoisomers), as a pale yellow viscous oil, $R_{\mathrm{F}} 0.38$ (50\% diethyl ether in petrol); 
$[\alpha]^{18}{ }_{\mathrm{D}}+16\left(c 0.55, \mathrm{CH}_{2} \mathrm{Cl}_{2}\right) ; v_{\max } / \mathrm{cm}^{-1}$ (neat) $3061 \mathrm{~s}, 3027 \mathrm{~s}, 2924 \mathrm{~s}, 2858 \mathrm{~s}, 1779 \mathrm{~s}, 1693$ s, 1641 w, 1495 m, 1454 m, 1385 s, 1347 m, 1209 m, 1107 m, 749 m and 699 s $\delta_{\mathrm{H}}(500$ $\mathrm{MHz}, \mathrm{CDCl}_{3}$, signals for 15a) 7.34-7.08 $(15 \mathrm{H}, \mathrm{m}), 6.56(1 \mathrm{H}, \mathrm{d}, J 16.0), 6.12(1 \mathrm{H}, \mathrm{dd}, J$ 16.0, 7.5), 5.78 (1 H, dt, J 17.0, 10.0), 5.07-5.02 (2 H, m), 4.74 (1 H, t J 7.5), 4.59 (1 H, m), $4.36(1 \mathrm{H}, \mathrm{t}, J$ 8.0), $4.25(1 \mathrm{H}, \mathrm{dt}, J$ 8.0, 4.5), 4.10-4.04 (2 H, m), $3.31(1 \mathrm{H}, \mathrm{m}), 3.18$ $(1 \mathrm{H}, \mathrm{dd}, J$ 13.5, 3.0), $2.82(1 \mathrm{H}, \mathrm{m}), 2.62(1 \mathrm{H}, \mathrm{m}), 2.60(1 \mathrm{H}, \mathrm{dd}, 13.5,10.0), 2.01(1 \mathrm{H}$, $\mathrm{m})$ and $1.91(1 \mathrm{H}, \mathrm{m})$; signals for $\mathbf{1 5 b}$ visible at $6.17(1 \mathrm{H}, \mathrm{dd}, J 16.0,7.5), 4.49(1 \mathrm{H}, \mathrm{m})$ and $3.06(1 \mathrm{H}, \mathrm{q}, J 10.0) ; \delta_{\mathrm{C}}\left(75 \mathrm{MHz}, \mathrm{CDCl}_{3}\right) 173.6,153.4,142.1,137.0,135.9,135.5$, $132.9,129.9,129.4,129.0,128.88,128.81,128.2,127.9,127.6,127.1,126.3,118.1,83.0$, 82.7, 66.3, 56.2, 55.9, 52.7, 38.4, 37.2 and 32.9; $\mathrm{m} / \mathrm{z}$ (ES+) 522 (60\%), 508 (37), 507 (35, $\left.[\mathrm{M}+\mathrm{H}]^{+}\right), 506(100), 404(78), 392(31)$ and $374(33)$; HRMS (ES+) found $[\mathrm{M}+\mathrm{Na}]^{+}$ 530.2294, $\mathrm{C}_{33} \mathrm{H}_{33} \mathrm{NO}_{4} \mathrm{Na}$ requires 530.2307.

(4S, 2'S, 3'S, 4'S, 5'S)-4-benzyl-3-[2'-(3,4-dimethoxyphenyl)-5'-phenethyl-4'vinyltetrahydrofuran-3'-carbonyl]-oxazolidin-2-one 16a

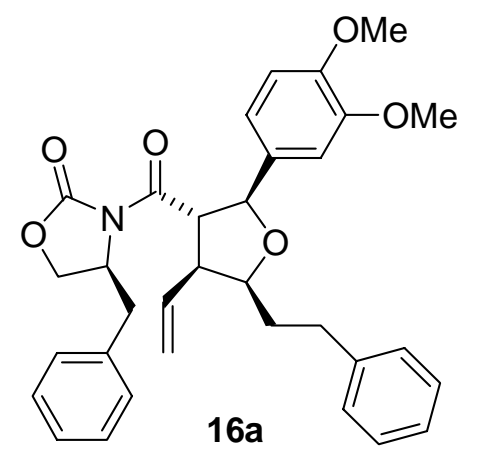

\section{Method 2 (Table 2, entry 6)}

Carried out according to the general method using trimethylsilyl trifluoromethanesulfonate $(5.5 \mu \mathrm{l}, 30 \mu \mathrm{mol}, 10 \mathrm{~mol} \%), 10(0.146 \mathrm{~g}, 0.303 \mathrm{mmol})$ and 3phenylpropionaldehyde $(40 \mu \mathrm{l}, 0.30 \mathrm{mmol})$ in dry dichloromethane $(7.0 \mathrm{ml})$ at $-78{ }^{\circ} \mathrm{C}$. The reaction mixture was stirred for $5 \frac{3}{4}$ hours at $-78^{\circ} \mathrm{C}$. Work-up and high performance liquid chromatography eluting with gradient elution: 35:65 to 95:5 acetonitrile-water, gave 16a $(0.068 \mathrm{~g}, 41 \%)$ as a pale yellow oil; $R_{\mathrm{F}} 0.38$ (50\% ethyl acetate in hexane); $[\alpha]^{18}+74\left(c 0.40, \mathrm{CH}_{2} \mathrm{Cl}_{2}\right) ; v_{\max } / \mathrm{cm}^{-1}$ (neat) $3027 \mathrm{w}, 2935 \mathrm{~m}, 2862 \mathrm{w}, 1781 \mathrm{~s}, 1692 \mathrm{~s}$, 1639 w, 1602 w, 1517 s, 1162 m, 1108 m, 1050 m, 1028 s, 911 s, 732 s, 701 s and 647 m; 
$\delta_{\mathrm{H}}\left(500 \mathrm{MHz}, \mathrm{CDCl}_{3}\right) 7.32-7.18(10 \mathrm{H}, \mathrm{m}), 6.97(1 \mathrm{H}, \mathrm{s}), 6.94(1 \mathrm{H}, \mathrm{d}, J$ 8.0), $6.82(1 \mathrm{H}$, d, $J$ 8.0), 5.97 (1 H, dt, $J$ 17.5, 10.0), $5.18(1 \mathrm{H}, \mathrm{d}, J 17.5), 5.16(1 \mathrm{H}, \mathrm{d}, J 10.0), 5.14$ (1 H, d, $J$ 9.0), 4.68 (1 H t, $J$ 9.0), $4.59(1 \mathrm{H}, \mathrm{m}), 4.30(1 \mathrm{H}, \mathrm{m}), 4.05$ (1 H, dd, $J$ 9.0, 2.5), $3.99(1 \mathrm{H}, \mathrm{t}, J$ 9.0) $3.88(3 \mathrm{H}, \mathrm{s}), 3.85(3 \mathrm{H}, \mathrm{s}), 3.39(1 \mathrm{H}, \mathrm{q}, J$ 9.0), $3.24(1 \mathrm{H}, \mathrm{dd}, J 13.5$, 3.5), 2.91 (1 H, ddd, J 15.5, 10.5, 5.0), 2.69 (1 H, m), 2.64 (1 H, dd, J 13.5, 10.0), 1.99 (1 $\mathrm{H}, \mathrm{m})$ and $1.85(1 \mathrm{H}, \mathrm{m}) ; \delta_{\mathrm{C}}\left(75 \mathrm{MHz}, \mathrm{CDCl}_{3}\right) 173.2,153.1,149.4,142.4,135.5,132.1$, 129.8, 129.4, 128.9, 128.8, 127.8, 126.3, 119.7, 118.4, 111.4, 110.2, 84.5, 81.2, 66.2, $56.3,56.0,55.3,54.5,38.4,34.9$ and 32.9 (29 signals expected, 27 signals visible); $\mathrm{m} / \mathrm{z}$ $(\mathrm{ES}+) 564\left(52 \%,[\mathrm{M}+\mathrm{Na}]^{+}\right), 542\left(30,[\mathrm{M}+\mathrm{H}]^{+}\right), 524\left(72,[\mathrm{M}-\mathrm{OH}]^{+}\right), 404(73$, $\left[\mathrm{M}-\mathrm{C}_{6} \mathrm{H}_{3}\left(\mathrm{OCH}_{3}\right)_{2}\right]^{+}, \quad 366 \quad\left(54, \quad\left[\mathrm{M}-\mathrm{PhCH}_{2} \mathrm{CHCH}_{2} \mathrm{CO}_{2} \mathrm{~N}\right]^{+}\right), \quad 365 \quad(100$, $\left[\mathrm{M}-\mathrm{PhCH}_{2} \mathrm{CHCH}_{2} \mathrm{CO}_{2} \mathrm{~N}\right]^{+}$) and 347 (37); HRMS (ES+) found $[\mathrm{M}+\mathrm{H}]^{+}$542.2545, $\mathrm{C}_{33} \mathrm{H}_{36} \mathrm{NO}_{6}$ requires 542.2543 .

(4S, $\quad 2$ 'S, $\quad 3$ 'S, $\quad$ 4'S, 5'R)-4-benzyl-3-[2'-(3,4-dimethoxyphenyl)-5-phenyl-4'vinyltetrahydrofuran-3'-carbonyl]-oxazolidin-2-one 17a

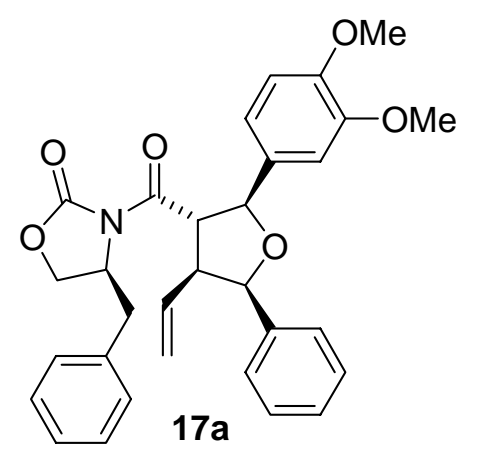

\section{Method 2 (Table 2, entry 7)}

Carried out according to the general method using trimethylsilyl trifluoromethanesulfonate $(5 \mu \mathrm{l}, 0.03 \mathrm{mmol}, 10 \mathrm{~mol} \%), \mathbf{1 0}(0.136 \mathrm{~g}, 0.283 \mathrm{mmol})$ and benzaldehyde $(29 \mu \mathrm{l}, 0.29 \mathrm{mmol})$ in dry dichloromethane $(6.5 \mathrm{ml})$ at $-78{ }^{\circ} \mathrm{C}$. The reaction mixture was stirred for $5 \frac{3}{4}$ hours at $-78{ }^{\circ} \mathrm{C}$. Work-up and high performance liquid chromatography eluting with gradient elution: 20:80 to 95:5 acetonitrile-water, gave 17a $(0.049 \mathrm{~g}, 34 \%)$ as a pale yellow oil, $R_{\mathrm{F}} 0.29\left(50 \%\right.$ EtOAc in petrol); $[\alpha]^{24}{ }_{\mathrm{D}}(+80 c 2.4$, $\mathrm{CH}_{2} \mathrm{Cl}_{2}$ ); $v_{\max } / \mathrm{cm}^{-1}$ (neat) 3063 w, 3029 w, 2960 w, 2934 w, 1779 s, 1690 s, 1639 w, 1594 w, 1518 s, 1454 m, 1386 s, 1264 s, 1027 s and 700 m; $\delta_{\mathrm{H}}\left(500 \mathrm{MHz}, \mathrm{CDCl}_{3}\right) 7.36-$ $7.18(10 \mathrm{H}, \mathrm{m}), 7.11(1 \mathrm{H}, \mathrm{d}, J 1.5), 7.07(1 \mathrm{H}, \mathrm{dd}, J$ 8.0, 1.5), 6.86 (1 H, d, $J$ 8.0), 5.42 (1 
$\mathrm{H}, \mathrm{d}, J$ 9.5), $5.34(1 \mathrm{H}, \mathrm{dt}, J 17.0,9.5), 5.29(1 \mathrm{H}, \mathrm{d}, J$ 9.5), $5.05(1 \mathrm{H}, \mathrm{d}, J 17.0), 4.91$ (1 $\mathrm{H}, \mathrm{d}, J$ 9.5) $4.90(1 \mathrm{H}, \mathrm{t}, J$ 9.5), $4.62(1 \mathrm{H}, \mathrm{m}), 4.05(1 \mathrm{H}, \mathrm{dd}, J$ 9.0, 2.5), $4.00(1 \mathrm{H}, \mathrm{m})$ $3.91(3 \mathrm{H}, \mathrm{s}), 3.87(3 \mathrm{H}, \mathrm{s}), 3.70(1 \mathrm{H} \mathrm{q}, J$ 9.5), $3.25(1 \mathrm{H}, \mathrm{dd}, J 13.5,3.0)$ and $2.63(1 \mathrm{H}$, dd, $J$ 13.5, 10.0); $\delta_{\mathrm{C}}\left(75 \mathrm{MHz}, \mathrm{CDCl}_{3}\right) 173.0,153.1,149.6,149.5,139.6,135.5,131.1$, 129.8, 129.4, 128.6, 128.0, 127.8, 127.5, 119.8, 117.7, 111.5, 110.6, 84.6, 83.6, 66.1, 57.0, 56.3, 56.0, 53.6 and 38.5 (27 signals expected, 25 signals visible); m/z (ES+) 514 $\left(17 \%,[\mathrm{M}+\mathrm{H}]^{+}\right), 497\left(34,[\mathrm{M}-\mathrm{OH}]^{+}\right), 496\left(85,[\mathrm{M}-\mathrm{OH}]^{+}\right), 376\left(50,\left[\mathrm{M}-\mathrm{C}_{6} \mathrm{H}_{4}\left(\mathrm{OCH}_{3}\right)_{2}\right]^{+}\right)$, 358 (51), 348 (64), 337 (100, [M-PhCH$\left.\left.{ }_{2} \mathrm{CH}\left(\mathrm{CH}_{2}\right) \mathrm{CO}_{2} \mathrm{~N}\right]^{+}\right)$and 319 (76); HRMS (ES+) found $[\mathrm{M}+\mathrm{H}]^{+} 514.2220, \mathrm{C}_{31} \mathrm{H}_{32} \mathrm{NO}_{6}$ requires 514.2230.

Preparation of (4S 2'S, 3'S, 4'S, 5'R) and (4S 2'S, 3'S, 4'S, 5'S)-3-[5'benzo[1,3]dioxol-5-yl-2'-(3',4'-dimethoxyphenyl)-4' -vinyltetrahydrofuran-3'carbonyl]-4-benzyloxazolidin-2-one 18a and $18 b$
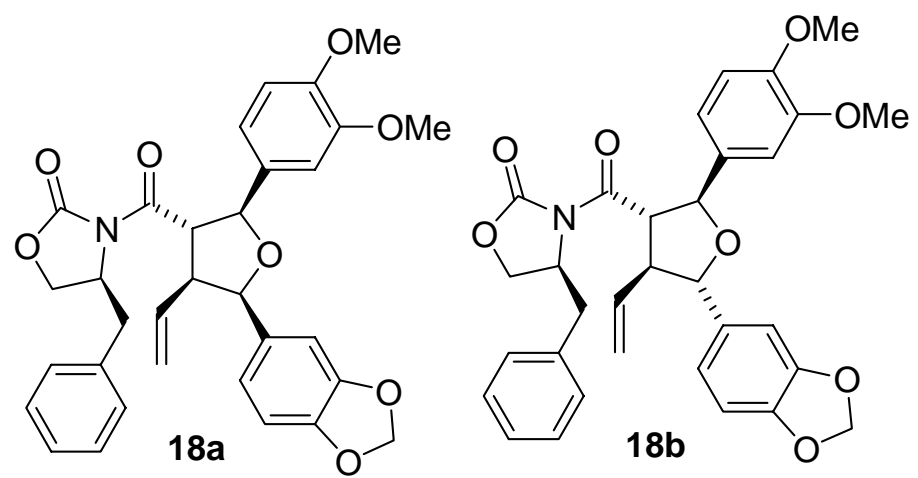

Method 2 (Table 2, entry 8)

Carried out according to the general method using trimethylsilyl trifluoromethanesulfonate $(6 \mu \mathrm{l}, 0.03 \mathrm{mmol}, 10 \mathrm{~mol} \%), 10(0.15 \mathrm{~g}, 0.32 \mathrm{mmol})$ and piperonal $(0.049 \mathrm{~g}, 0.33 \mathrm{mmol})$ in dry dichloromethane $(7.5 \mathrm{ml})$ at $-78{ }^{\circ} \mathrm{C}$. The reaction mixture was stirred for $6 \frac{3}{4}$ hours at $-78{ }^{\circ} \mathrm{C}$. Work-up and high performance flash chromatography eluting with gradient elution: 20:80 to 95:5 acetonitrile-water, gave 18a and $\mathbf{1 8 b}(0.11 \mathrm{~g}, 63 \%$ as a $91: 9$ mixture of diastereoisomers), as a pale yellow viscous liquid, $R_{\mathrm{F}} 0.30$ (50\% diethyl ether in petrol); $[\alpha]^{25}+52\left(c 1.3, \mathrm{CH}_{2} \mathrm{Cl}_{2}\right) ; v_{\max } / \mathrm{cm}^{-1}$ (neat) 2994 w, 2956 m, 2922 s, 2851 m, 1778 s, 1691 s, 1640 w, 1607 w, 1517 s, 1504 s, 1490 s, $1462 \mathrm{~m}, 1445 \mathrm{~s}, 1386 \mathrm{~s}, 1350 \mathrm{~m}, 1261 \mathrm{~s}, 1239 \mathrm{~s}, 1103 \mathrm{~m}, 1035 \mathrm{~s}, 930 \mathrm{~m}$ and $701 \mathrm{~s} ; \delta_{\mathrm{H}}$ (500 MHz, $\mathrm{CDCl}_{3}$, signals for 18a); 7.33-6.78 $(11 \mathrm{H}, \mathrm{m}), 5.97(2 \mathrm{H}, \mathrm{s}), 5.40(1 \mathrm{H}, \mathrm{dt}, J$ 
17.0, 9.5), $5.33(1 \mathrm{H}, \mathrm{d}, J$ 9.5), $5.24(1 \mathrm{H}, \mathrm{d}, J 9.5), 5.06(1 \mathrm{H}, \mathrm{d}, J 17.0), 4.94(1 \mathrm{H}, \mathrm{d}, J$ 9.5), $4.87(1 \mathrm{H}, \mathrm{t}, J$ 9.5), $4.61(1 \mathrm{H}, \mathrm{m}), 4.06(1 \mathrm{H}, \mathrm{dd}, J$ 9.0, 2.5), $3.99(1 \mathrm{H}, \mathrm{t}, J$ 9.0), $3.91(3 \mathrm{H}, \mathrm{s}), 3.88(3 \mathrm{H}, \mathrm{s}), 3.64(1 \mathrm{H}, \mathrm{q}, J$ 9.5), $3.26(1 \mathrm{H}, \mathrm{dd}, J$ 13.5, 10.0), $2.63(1 \mathrm{H}, \mathrm{dd}$, $J$ 13.5, 9.5); signals for $\mathbf{1 8 b}$ visible at $5.98(2 \mathrm{H}, \mathrm{s}), 5.83(1 \mathrm{H}$, ddd, $J$ 17.0, 10.0, 8.0), $5.13\left(1 \mathrm{H}, \mathrm{d}, J\right.$ 10.0), $4.73(1 \mathrm{H}, \mathrm{t}, J 9.0)$ and $3.06(1 \mathrm{H}, \mathrm{dd}, J 13.5,3.0)$; $\delta_{\mathrm{C}}(75 \mathrm{MHz}$, $\left.\mathrm{CDCl}_{3}\right)$ 172.9, 153.1, 149.6, 149.5, 148.0, 136.3, 135.5, 133.7, 131.0, 129.8, 129.4, 129.4, 129.3, 127.8, 120.8, 119.9, 117.7, 111.5, 110.5, 108.4, 101.4, 84.5, 83.5, 77.6, 66.2, 57.1, 56.3, 53.5, 38.5 (30 signals expected, 29 signals seen); signals for $\mathbf{1 8 b}$ visible at 135.7, $121.1,120.2,118.9,110.9,110.7,107.6,85.6,81.6,66.4$ and 54.1; $\mathrm{m} / \mathrm{z}(\mathrm{ES}+) 541$ (45\%, $\left.[\mathrm{M}-\mathrm{OH}]^{+}\right), 540\left(100,[\mathrm{M}-\mathrm{OH}]^{+}\right), 418(55), 402(30), 392$ (58) and 363 (90); HRMS $(\mathrm{ES}+)$ found $[\mathrm{M}+\mathrm{Na}]^{+}$580.1971, $\mathrm{C}_{32} \mathrm{H}_{31} \mathrm{NONa}$ requires 580.1947 .

\section{$\left(4 S, \quad 2^{\prime} S, \quad 3^{\prime} R, 4^{\prime} R, 5^{\prime} R\right)$-[5'-benzo[1,3]dioxol-5-yl-2'-(3',4'-dimethoxyphenyl)-4'-} hydroxymethytetrahydrofuran-3'-yl]-methanol 19

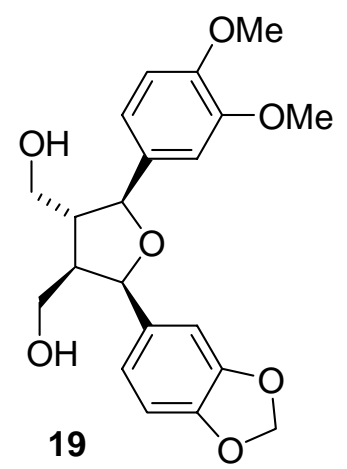

Water $(0.22 \mathrm{ml})$, 4-methylmorpholine $N$-oxide $(0.039 \mathrm{~g}, 0.33 \mathrm{mmol})$ and potassium osmate dihydrate ( $3 \mathrm{mg}, 0.009 \mathrm{mmol}, 5 \mathrm{~mol} \%$ ) were sequentially added to a solution of 18 (0.108 g, $194 \mathrm{mmol}, 91: 9$ diastereomeric mixture) in dry THF (2.2 ml). The reaction mixture was stirred for 40 hours at room temperature. Water $(5 \mathrm{ml})$ was added to the reaction mixture which was then extracted with dichloromethane $(5 \times 10 \mathrm{ml})$. The combined organic extracts were dried $\left(\mathrm{MgSO}_{4}\right)$, filtered and evaporated under reduced pressure to give the crude diol. The crude diol was dissolved in methanol ( $2 \mathrm{ml})$ followed by addition of a solution sodium metaperiodate $(0.051 \mathrm{~g}, 0.024 \mathrm{mmol})$ in methanol-water $(3.2: 1,3.8 \mathrm{ml})$. The reaction mixture was stirred for $3 \frac{1}{2}$ hours at room temperature. The reaction mixture was filtered and the residue washed with dichloromethane. Water $(5 \mathrm{ml})$ was added to the organic filtrate and the aqueous layer was extracted with 
dichloromethane $(4 \times 20 \mathrm{ml})$. The combined organic extracts were dried $\left(\mathrm{MgSO}_{4}\right)$, filtered and evaporated under reduced pressure to give the crude aldehyde. The crude aldehyde was dissolved in THF $(1.5 \mathrm{ml})$ followed by the addition of a solution of sodium borohydride $(0.045 \mathrm{~g}, 0.98 \mathrm{mmol})$ in water $(0.8 \mathrm{ml})$. The reaction mixture was stirred for $14^{3} / 4$ hours at room temperature. The reaction mixture was quenched with aqueous $1 \mathrm{M}$ hydrochloric acid solution $(0.4 \mathrm{ml})$ then extracted with dichloromethane $(3 \times 20 \mathrm{ml})$. The combined organic extracts were dried $\left(\mathrm{MgSO}_{4}\right)$, filtered and evaporated under reduced pressure to give the crude product which was purified by flash chromatography gradient elution: 40:60-70:30 ethyl acetate-petrol, to give $\mathbf{1 9}(0.036 \mathrm{~g}, 48 \%$ as a 74:26 mixture of diastereoisomers), as a pale yellow oil, $R_{\mathrm{F}} 0.21$ (ethyl acetate); $[\alpha]^{24}{ }_{\mathrm{D}}+14(c 0.55$, $\mathrm{CH}_{2} \mathrm{Cl}_{2}$ ); $v_{\text {max }} / \mathrm{cm}^{-1}$ (neat) 3368 br, 2956 s, 2924 s, $2851 \mathrm{w}, 1608 \mathrm{~m}, 1593 \mathrm{~m}, 1516 \mathrm{~s}, 1503$ s, 1489 s, 1462 s, 1444 s, 1260 s, 1029 s, 932 w, and 809 m; $\delta_{\mathrm{H}}\left(300 \mathrm{MHz}, \mathrm{CDCl}_{3}\right)$; 7.03$6.77(6 \mathrm{H}, \mathrm{m}), 5.97(2 \mathrm{H}, \mathrm{s}), 5.12(1 \mathrm{H}, \mathrm{d}, J$ 8.5), $4.50(1 \mathrm{H}, \mathrm{d}, J$ 9.5), $3.92(3 \mathrm{H}, \mathrm{s}), 3.90(3$ $\mathrm{H}, \mathrm{s}), 3.78$ (1 H, dd, $J$ 10.5, 3.5), 3.63 (1 H, t, $J 10.5), 3.38$ (1 H, dd, $J$ 10.5, 4.5), 3.18 (1 $\mathrm{H}, \mathrm{t}, J 10.5), 2.60(1 \mathrm{H}, \mathrm{m}), 2.27(1 \mathrm{H}, \mathrm{m})$; signals for minor diastereoisomer visible at $5.98(2 \mathrm{H}, \mathrm{s}), 5.13(1 \mathrm{H}, \mathrm{d}, J 8.5), 3.88(3 \mathrm{H}, \mathrm{s})$ and $3.14(1 \mathrm{H}, \mathrm{t}, J 10.0)$; $\delta_{\mathrm{C}}(75 \mathrm{MHz}$, $\left.\mathrm{CDCl}_{3}\right)$ 148.1, 148.0, 146.7, 146.1, 131.8, 131.4, 118.8, 118.1, 110.1, 108.9, 107.1, 106.0, 100.1, 81.6, 80.2, 62.8, 62.1, 54.9, 54.2, 49.9 (21 signals expected, 20 signals seen); signals for minor diastereoisomer visible at 148.0, 147.5, 119.4, 117.6, 109.9, 108.6, 107.2, 103,6, 62.0, 54.1 and 49.7; m/z (ES+) $411\left(13 \%,[\mathrm{M}+\mathrm{Na}]^{+}\right), 353(100)$ and 335 (52); HRMS (ES+) found [M+Na] ${ }^{+} 411.1428, \mathrm{C}_{21} \mathrm{H}_{24} \mathrm{O}_{7} \mathrm{Na}$ requires 411.1420. 


\section{(+)-Virgatusin 1}

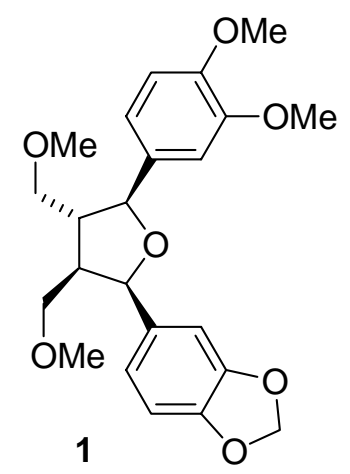

A solution of 19 (17 mg, $0.044 \mathrm{mmol}, 74: 26$ diastereomeric mixture) in dry THF (1.5 $\mathrm{ml}$ ) was added to a mixture of sodium hydride $(5.4 \mathrm{mg}, 60 \%$ dispersion in mineral oil, $0.14 \mathrm{mmol})$ in dry THF $(0.10 \mathrm{ml})$. The reaction mixture was stirred for $1 \frac{1}{2}$ at room temperature. Methyl iodide $(8.5 \mu 1,0.14 \mathrm{mmol})$ was added to the reaction mixture and the reaction mixture was stirred at room temperature for $20 \frac{1}{2}$ hours. The reaction mixture was diluted with diethyl ether, dried $\left(\mathrm{MgSO}_{4}\right)$, filtered and evaporated under reduced pressure to give the crude product which was purified by flash chromatography eluting with 60:40 ethyl acetate-petrol, to give 1 (9 $\mathrm{mg}, 50 \%)$ as a 74:26 mixture of diastereoisomers, as a pale yellow oil whose data agreed with that reported in the literature $^{1}$ for the enantiomeric compound. $R_{\mathrm{F}} 0.57$ (ethyl acetate); $[\alpha]^{22}{ }_{\mathrm{D}}+16$ (c 0.40 , $\mathrm{CH}_{2} \mathrm{Cl}_{2}$ ) (lit. ${ }^{78}[\alpha]^{25}{ }_{\mathrm{D}}-12.7\left(c 0.5, \mathrm{CH}_{2} \mathrm{Cl}_{2}\right) ; v_{\max } / \mathrm{cm}^{-1}$ (neat) $3434 \mathrm{w}, 2929 \mathrm{~s}, 1727 \mathrm{~s}, 1607$ m, 1516 m, 1491 m, 1445 m , 1382 w, 1269 s, 1234 s, 1193 w, 1124 m, 1073 w 1037 m, $937 \mathrm{w}, 809 \mathrm{w}$ and $741 \mathrm{w} ; \delta_{\mathrm{H}}\left(300 \mathrm{MHz}, \mathrm{CDCl}_{3}\right) 6.93-6.77(6 \mathrm{H}, \mathrm{m}), 5.96(2 \mathrm{H}, \mathrm{s}), 5.08(1$ H, d, J 7.5), 4.73 (1 H, d, J 8.0), 3.93 (3 H, s), 3.90 (3 H, s), $3.53(2 \mathrm{H}, \mathrm{m}), 3.36$ (3 H, s), $3.09(3 \mathrm{H}, \mathrm{s}), 3.08(1 \mathrm{H}, \mathrm{m}), 2.96(1 \mathrm{H}, \mathrm{m}), 2.61(1 \mathrm{H}, \mathrm{m}), 2.33(1 \mathrm{H}, \mathrm{m})$; signals for minor diastereoisomer visible at $5.97(2 \mathrm{H}, \mathrm{s}), 5.10(1 \mathrm{H}, \mathrm{m}), 4.71(1 \mathrm{H}, \mathrm{d}, J$ 7.5) and $3.08(3 \mathrm{H}, \mathrm{s}) ; \delta_{\mathrm{C}}\left(75 \mathrm{MHz}, \mathrm{CDCl}_{3}\right) 148.9,148.5,147.4,146.6,134.1,132.8,119.6,118.8$, 110.9, 109.6, 107.9, 107.1, 101.0, 82.6, 81.5, 73.1, 73.0, 59.1, 58.7, 55.92, 55.85, 50.9, 46.6; signals for minor diastereoisomer visible at 118.5, 110.7, 110.9, 108.1 and 51.1; m/z (ES+) 439 (47\%, [M+Na] $\left.]^{+}\right), 413$ (48), 367 (85) and 335 (100); HRMS (ES+) found $[\mathrm{M}+\mathrm{Na}]^{+} 439.1724, \mathrm{C}_{23} \mathrm{H}_{28} \mathrm{O}_{7} \mathrm{Na}$ requires 439.1733 .

\footnotetext{
${ }^{1}$ Huang, Y.-L.; Chen, C.-C.; Hsu, F.-L.; Chen, C.-F. J. Nat. Prod. 1996, 59, 520.
} 


\section{NMR SPECTRA}

Siloxane 9
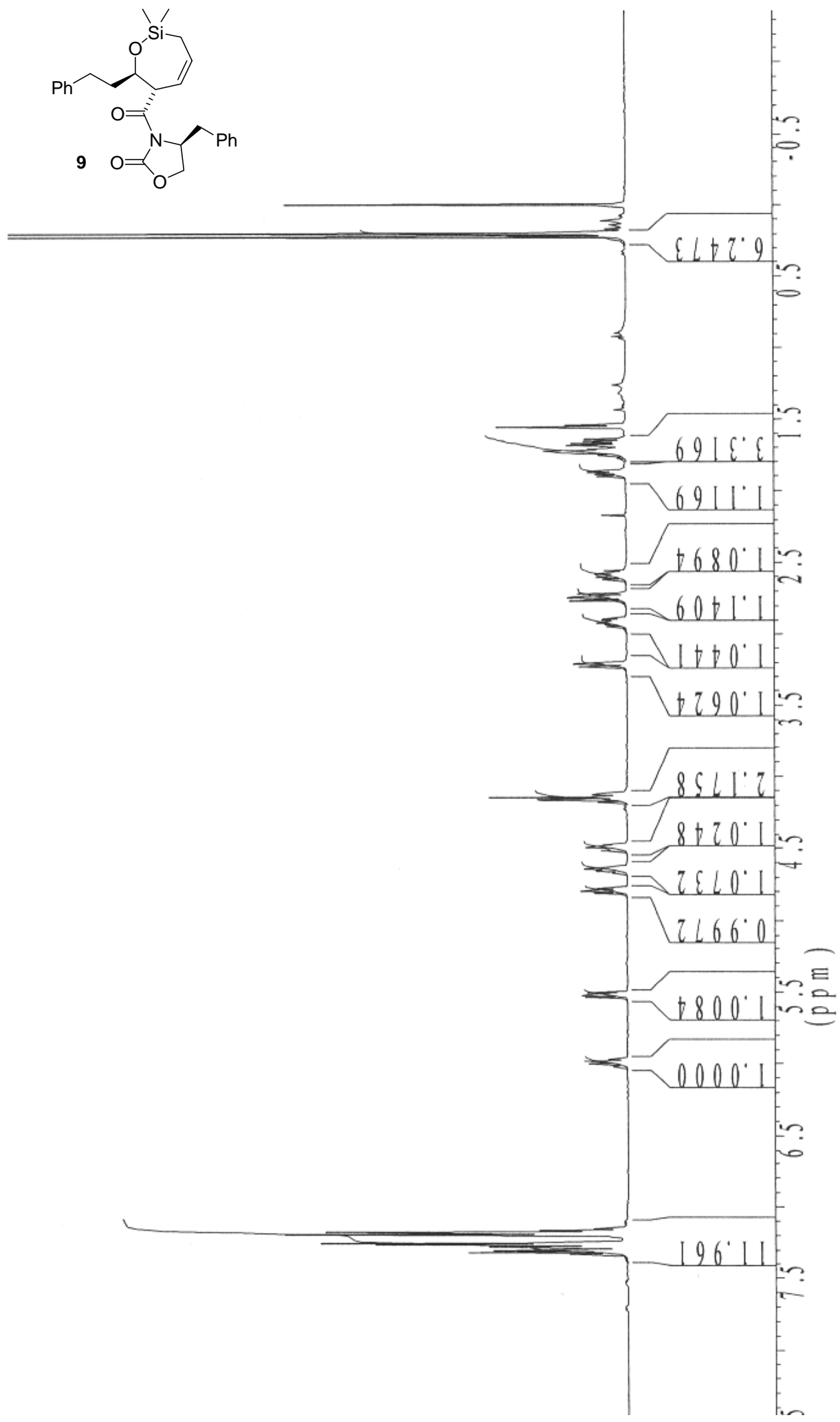
Siloxane 9
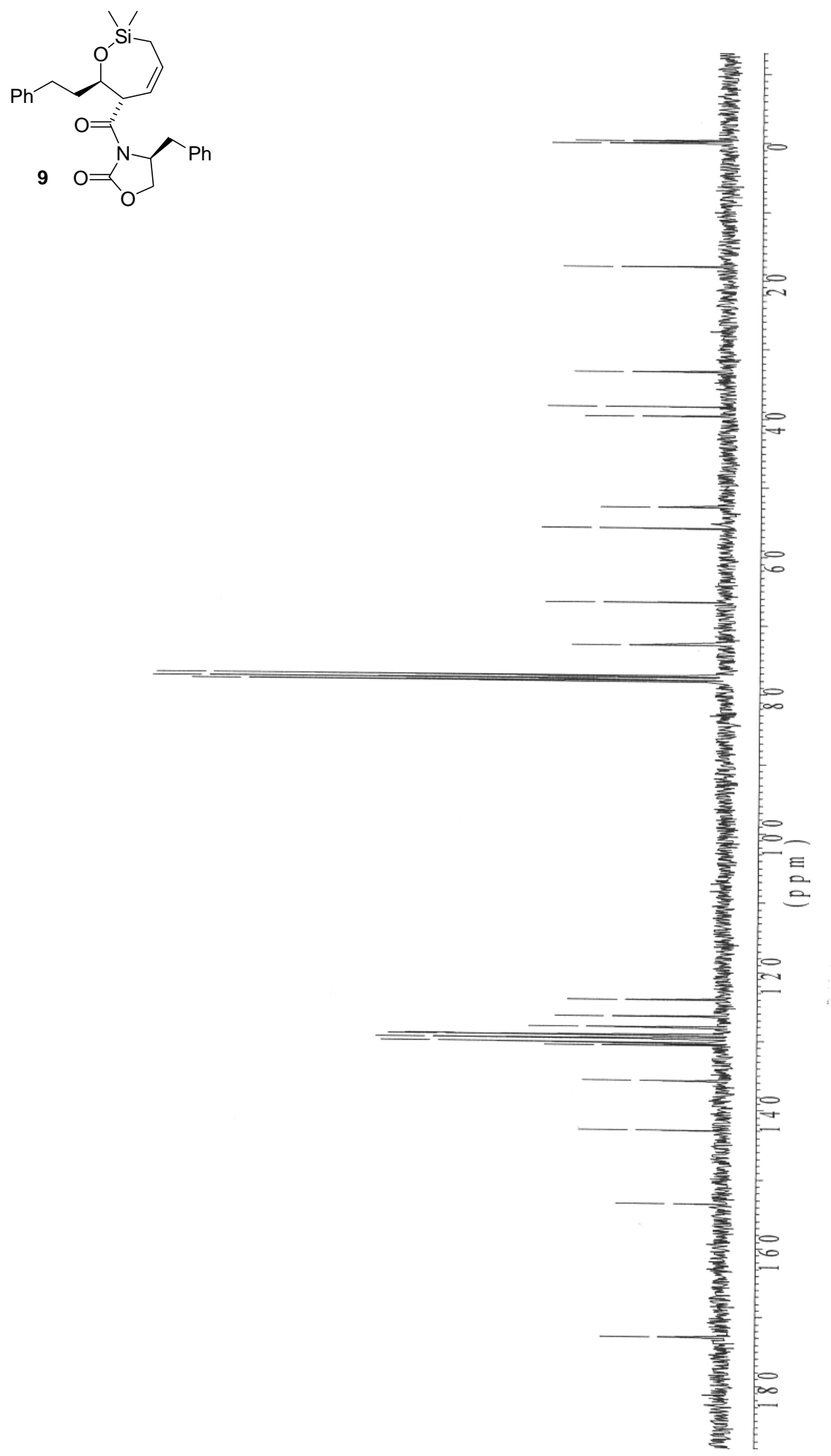


\section{Siloxane 10}

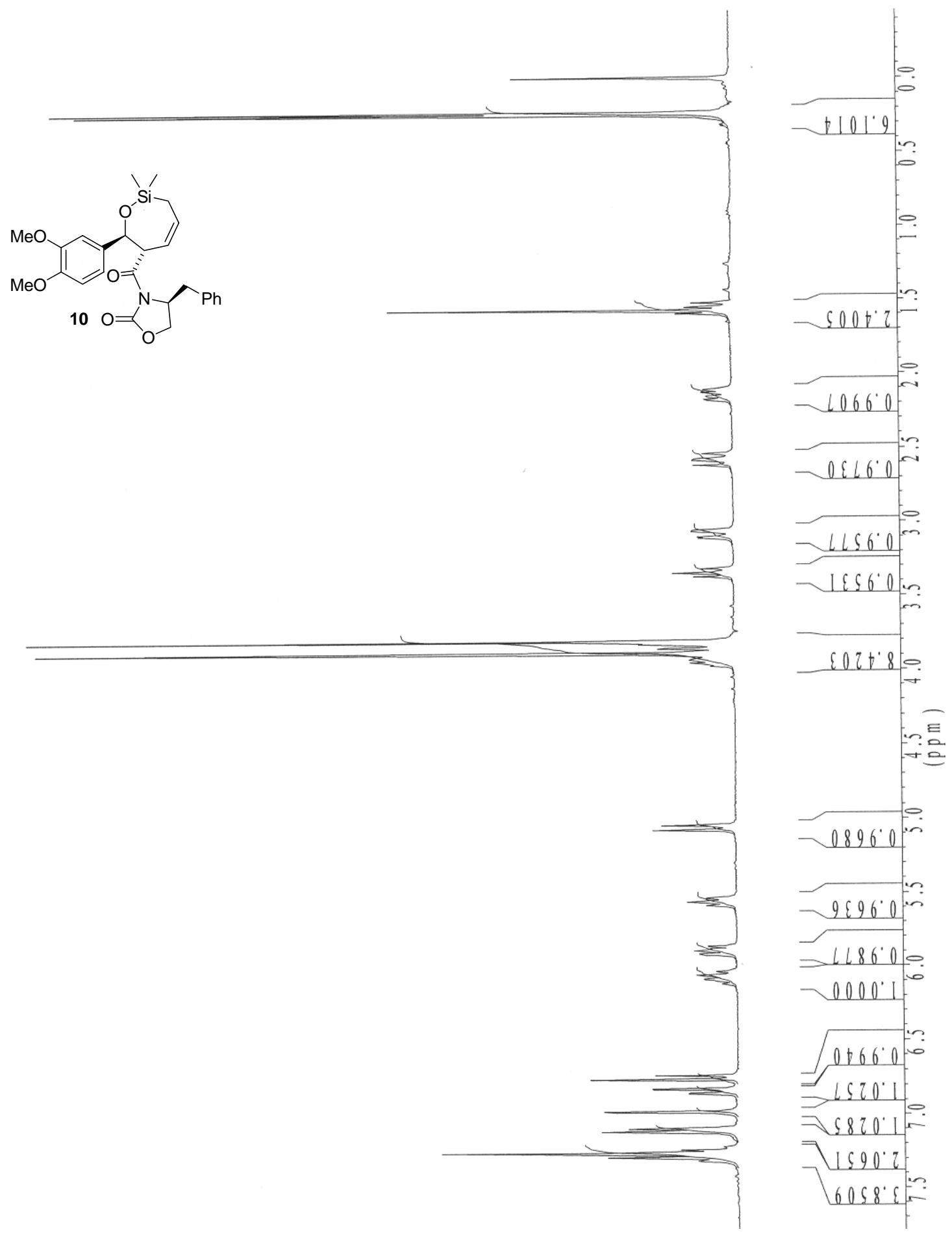


Siloxane 10
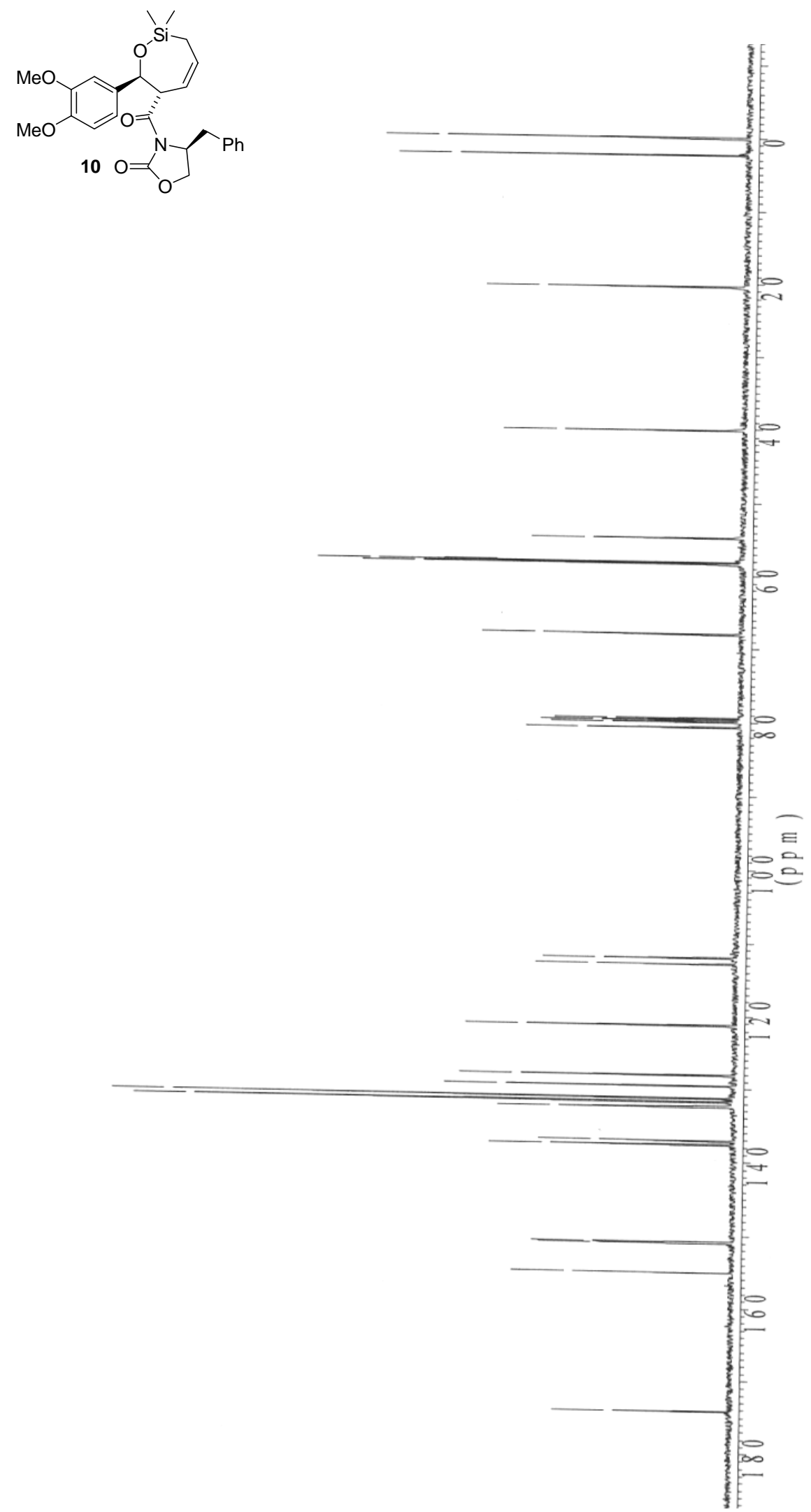
Compound 11a (Table 1, Entry 1; Table 2, Entry 2)
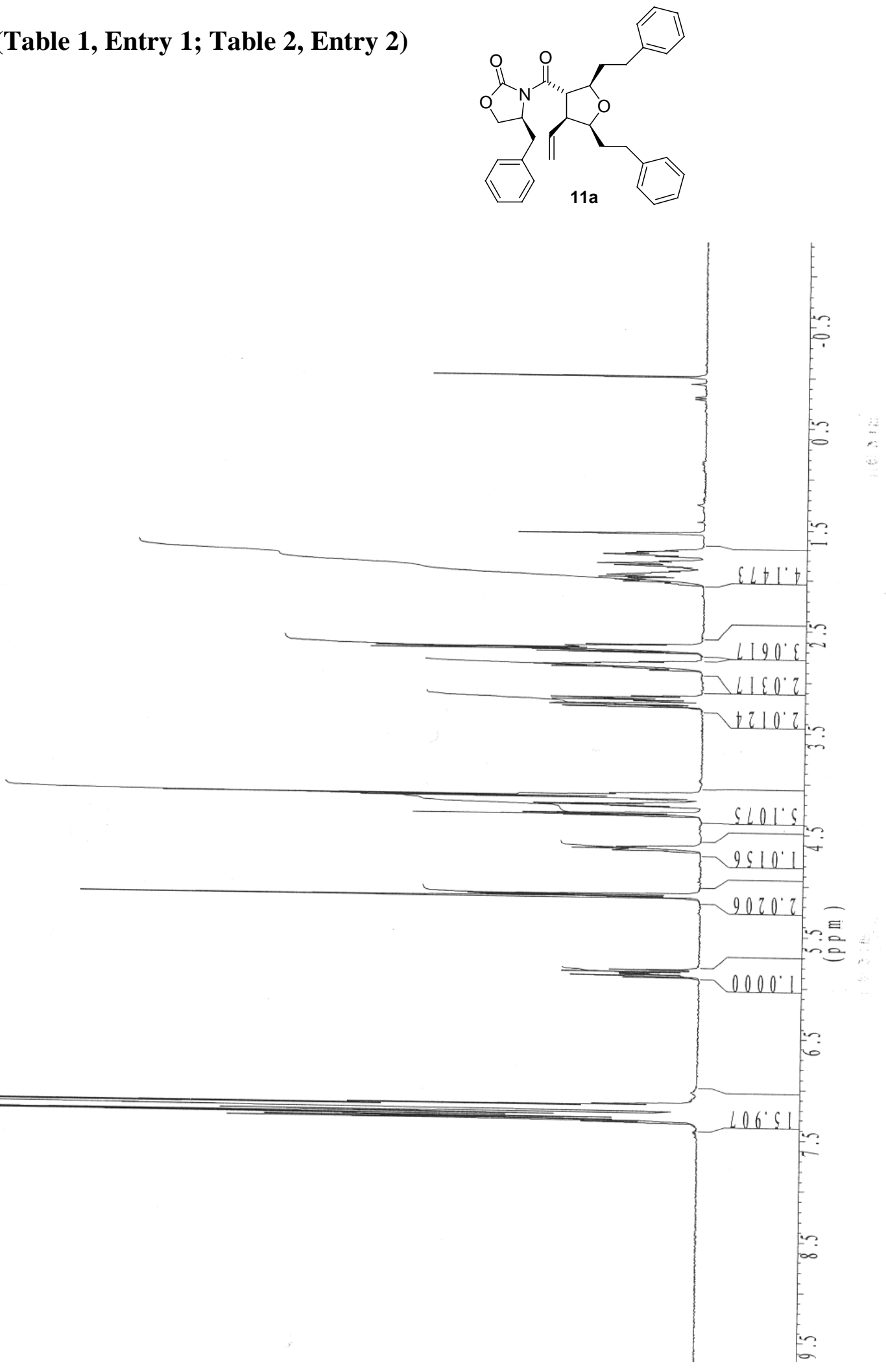
Compound 11a (Table 1, Entry 1; Table 2, Entry 2)
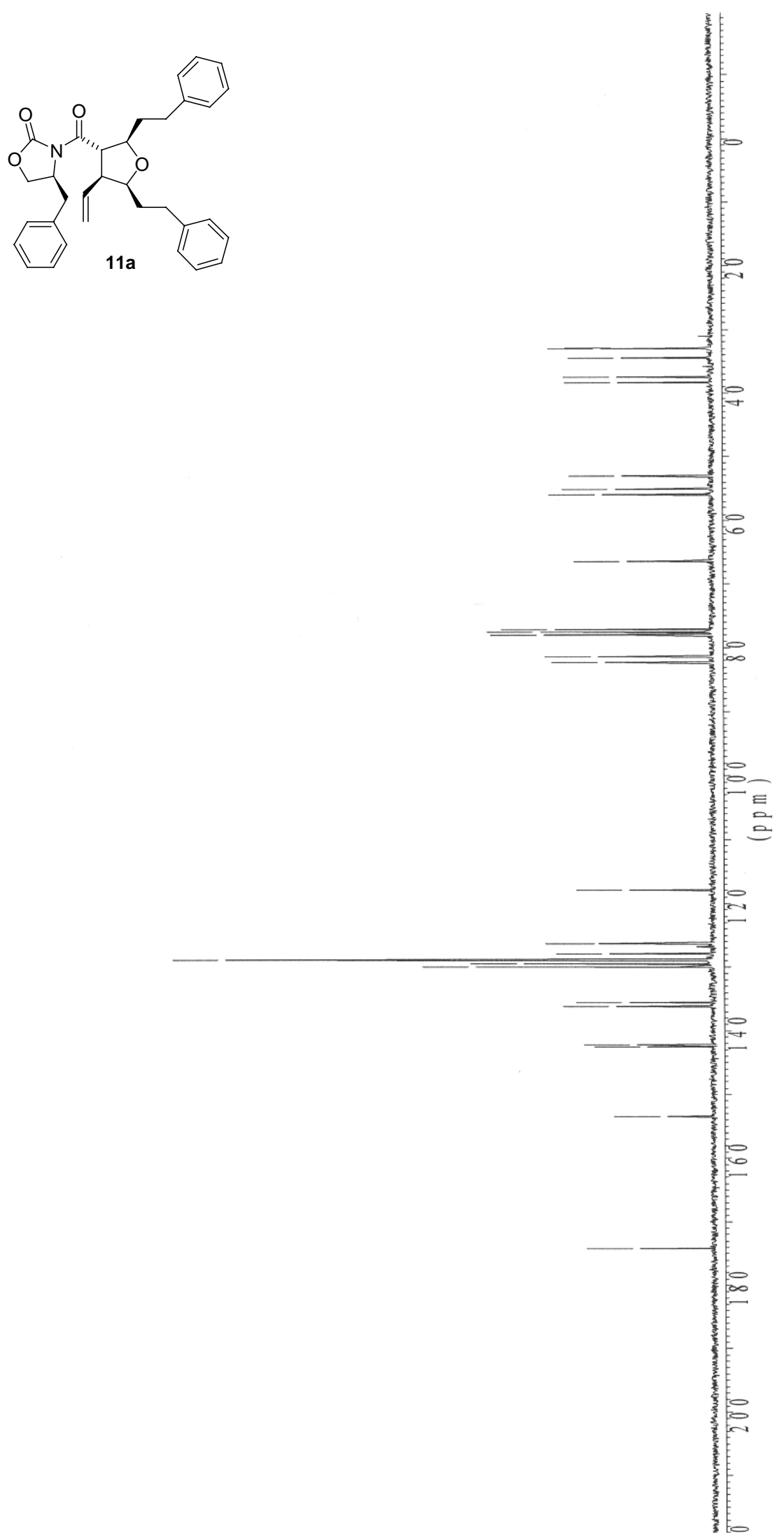
Compound 12a (Table 1, Entry 2; Table 2, Entry 2)
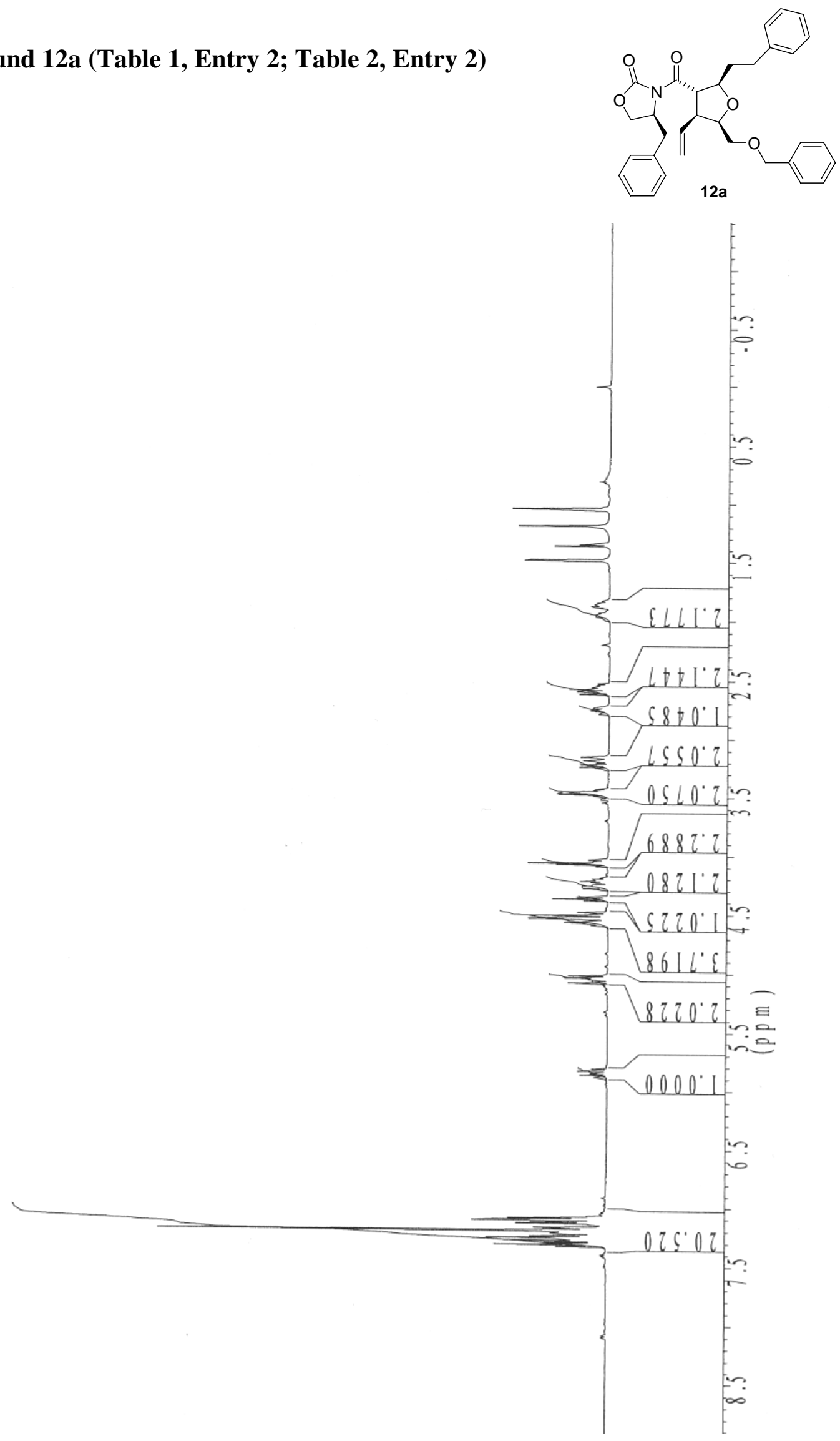
Compound 12a (Table 1, Entry 2; Table 2, Entry 2)
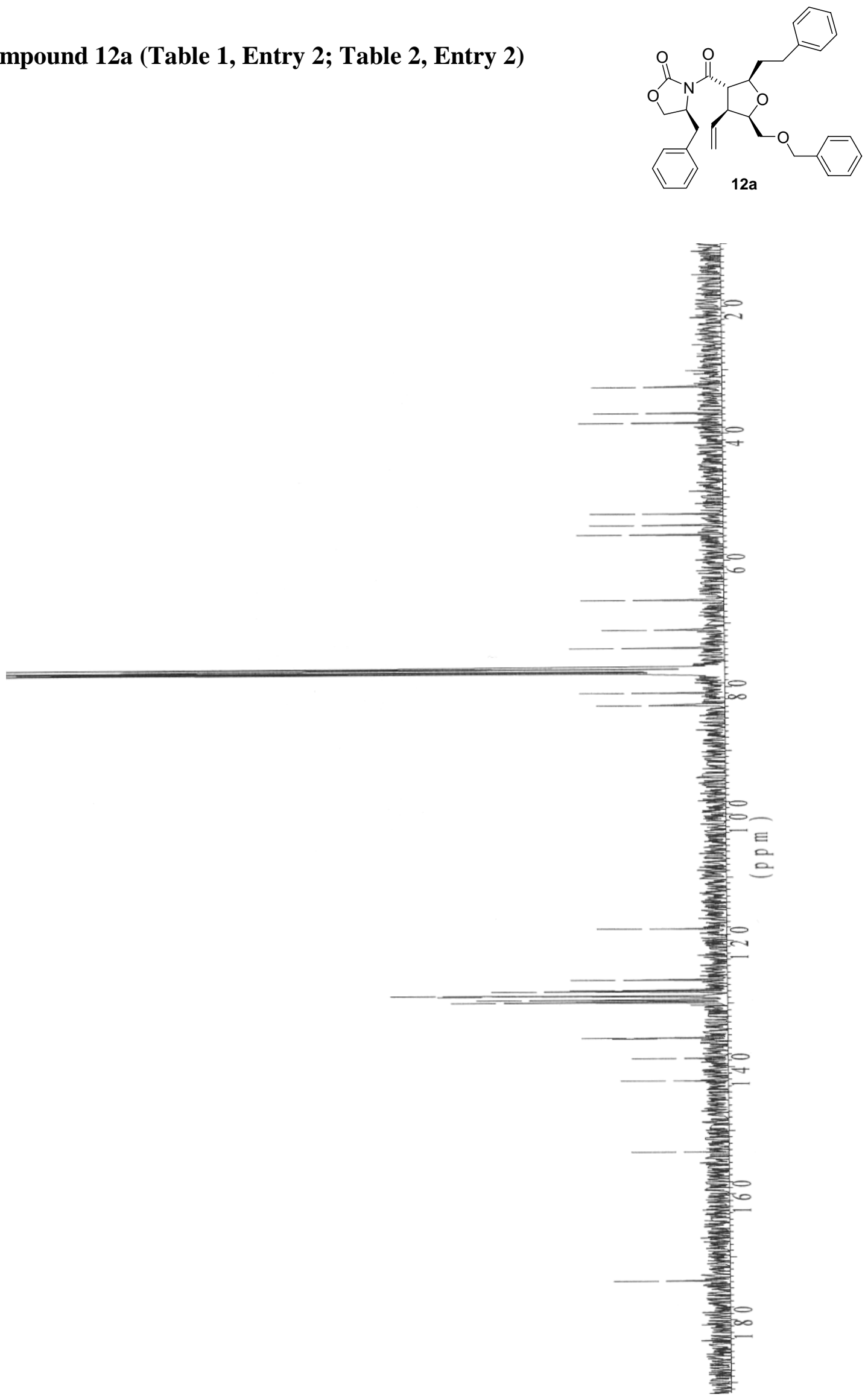
Compounds 13a/13b (93:7, Table 1, Entry 3)
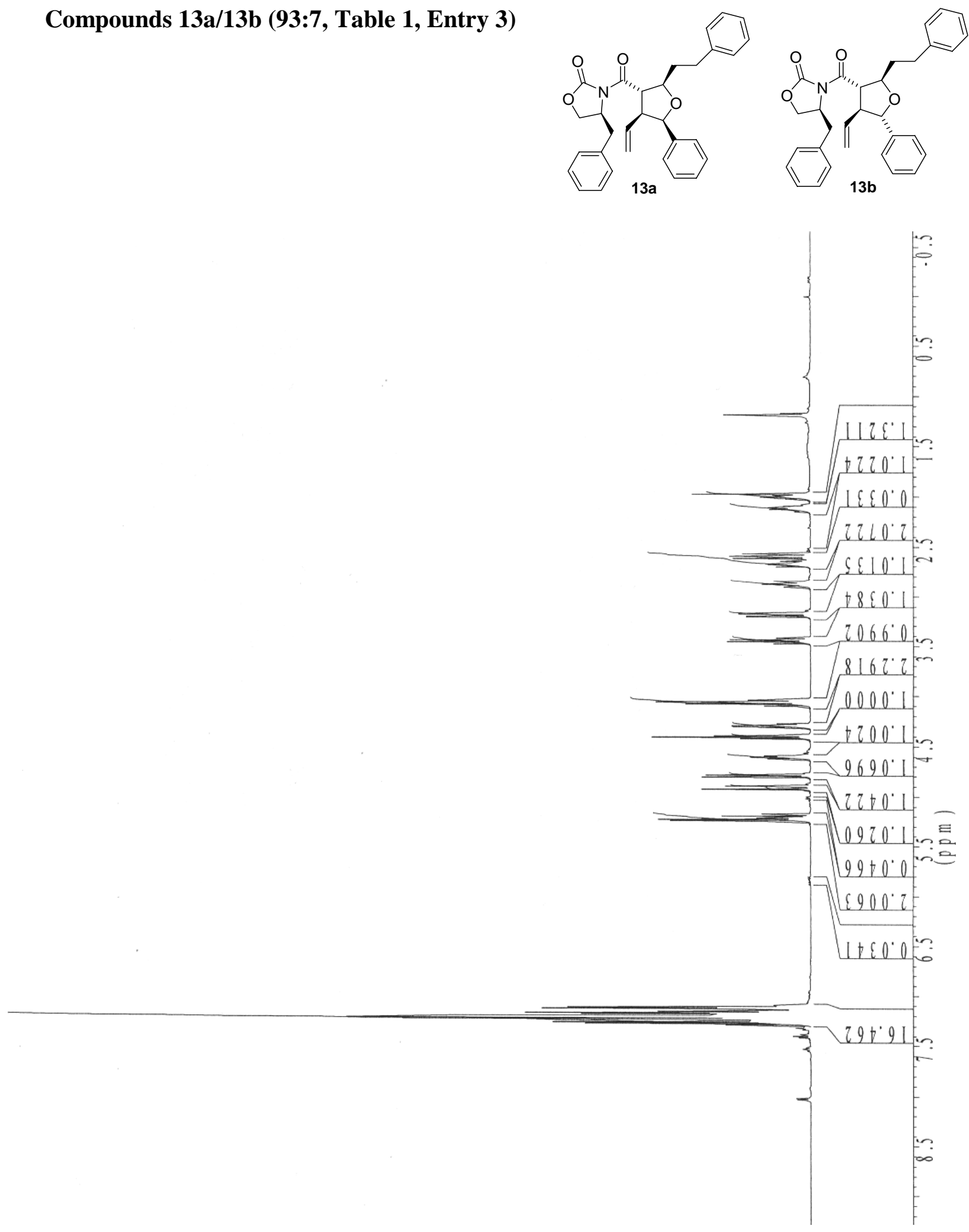


\section{Compounds 13a/13b (93:7, Table 1, Entry 3)}
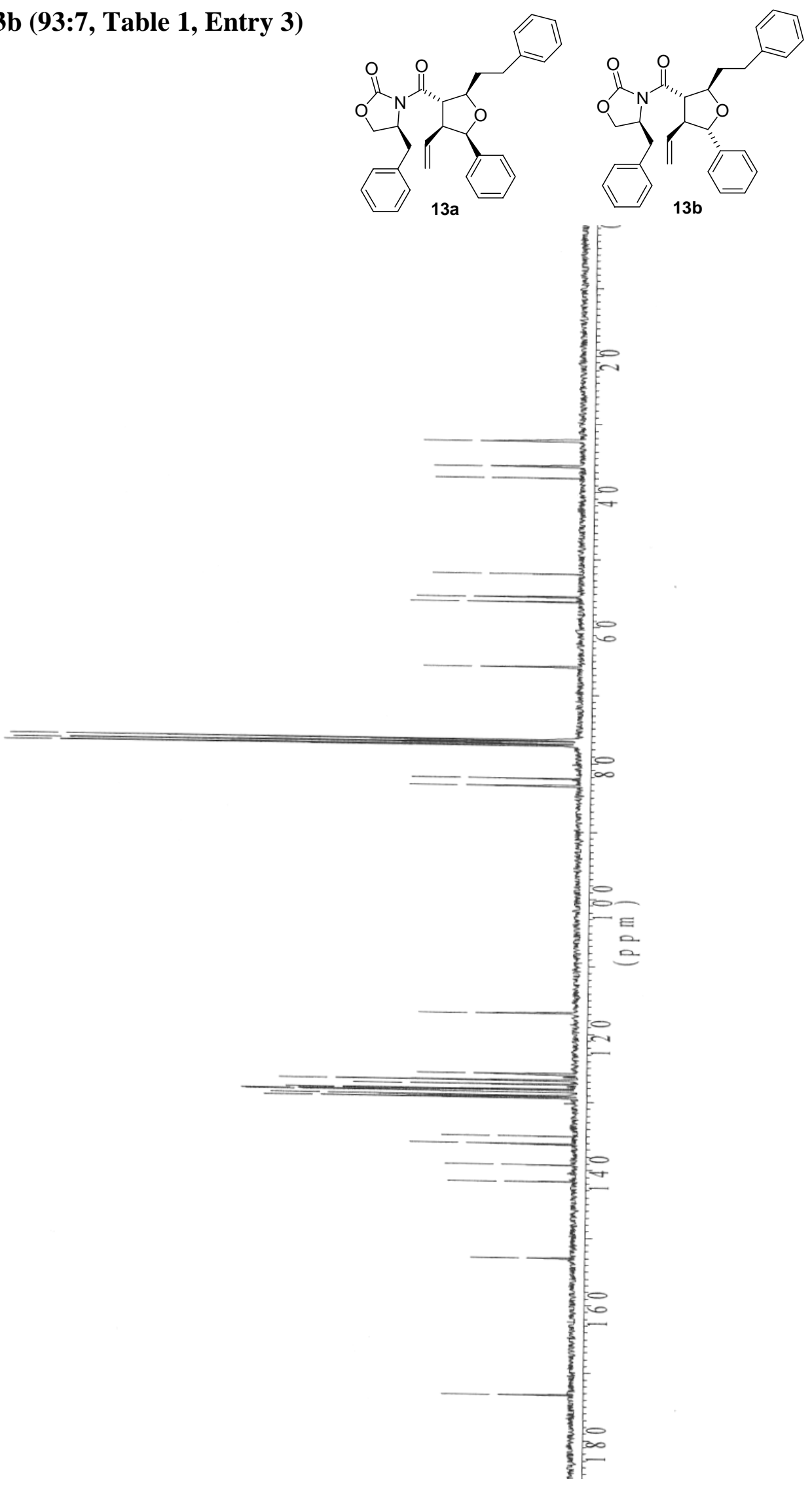
Compounds 13a/13b + 13c (unknown stereochemistry) (80:12:8, Table 1, Entry 4)
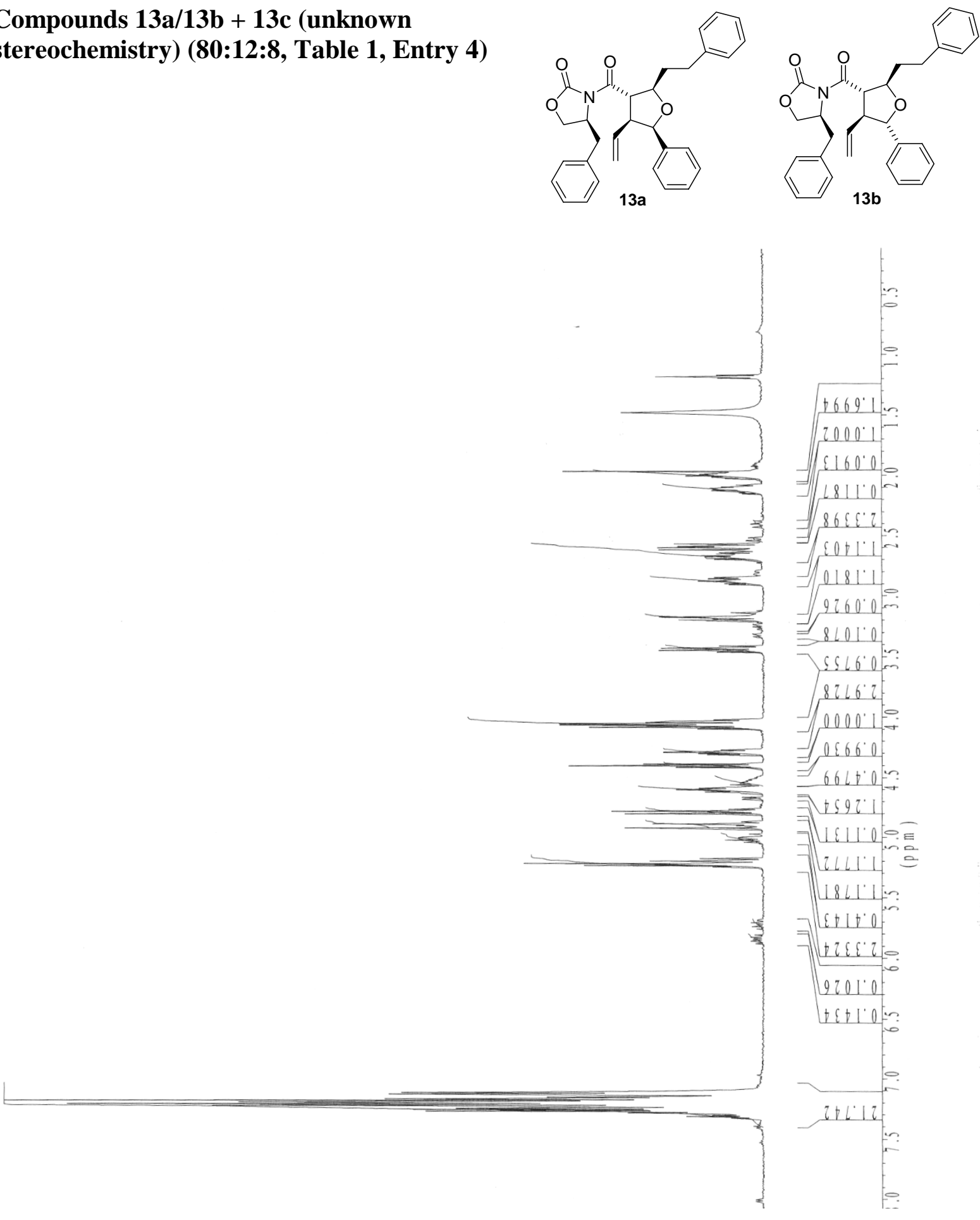
Compounds 14a/14b (9:91, Table 1, Entry 6)
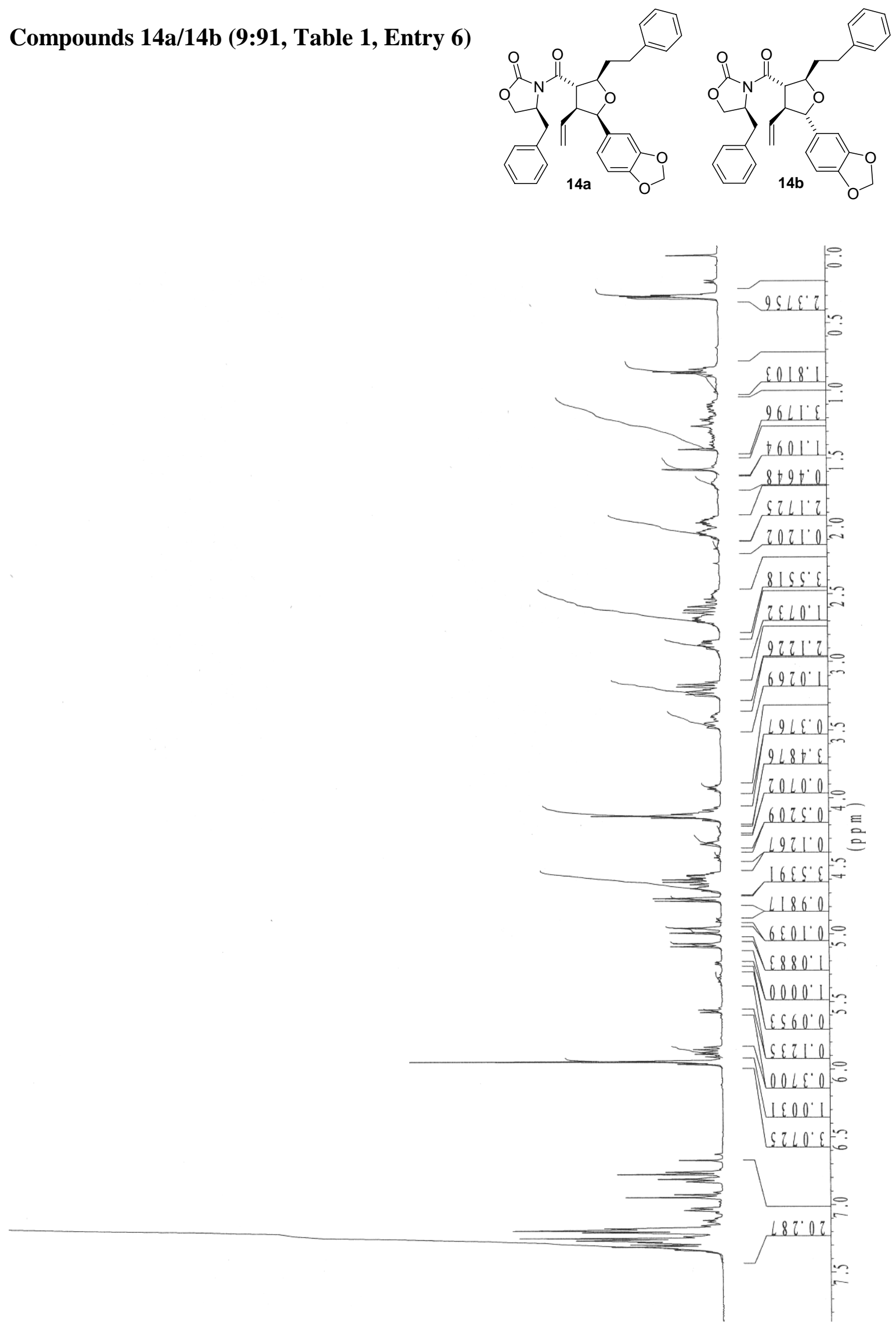
Compounds 14a/14b (9:91, Table 1, Entry 6)
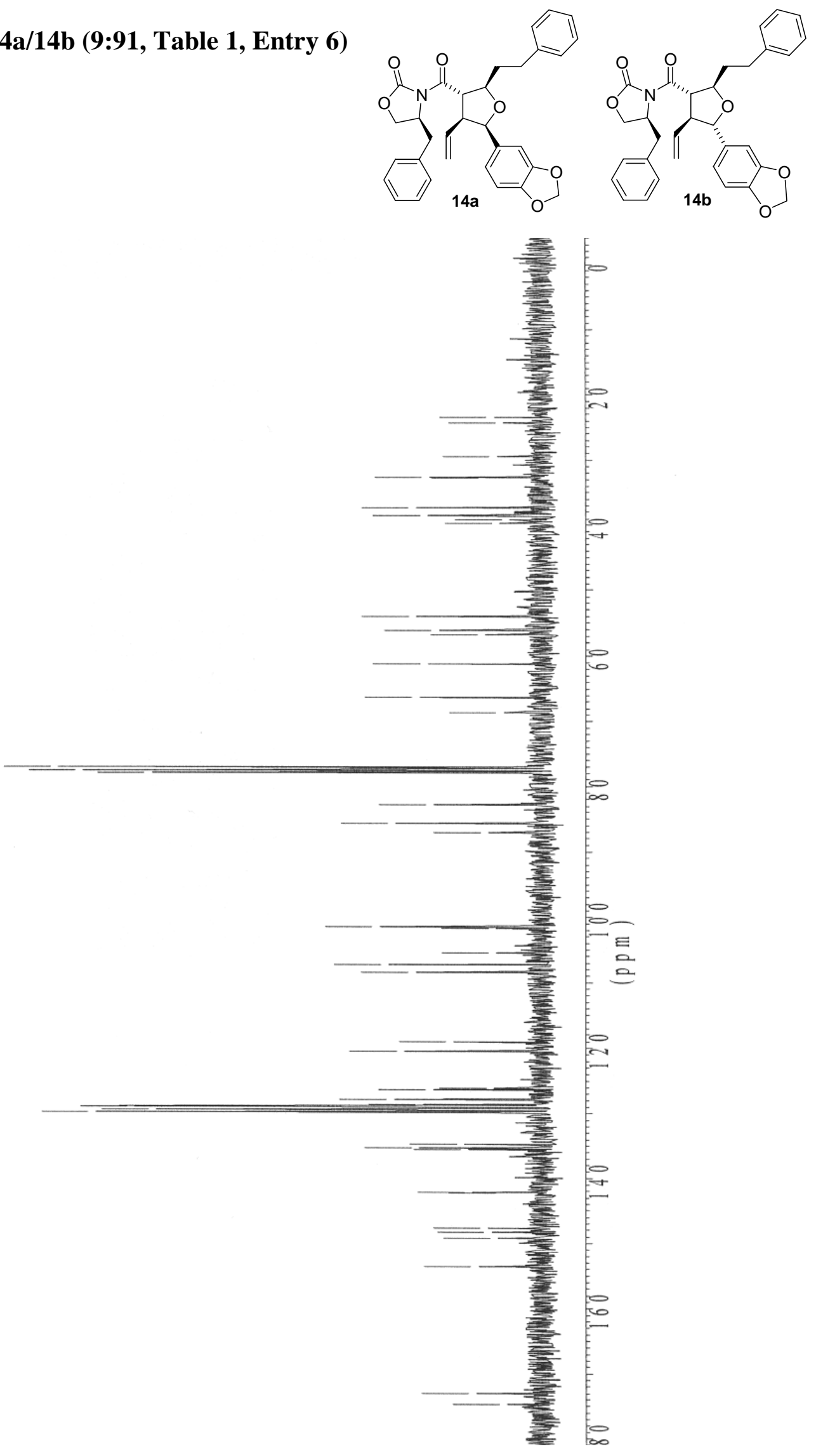
Compound 14a (Table 2, Entry 4)
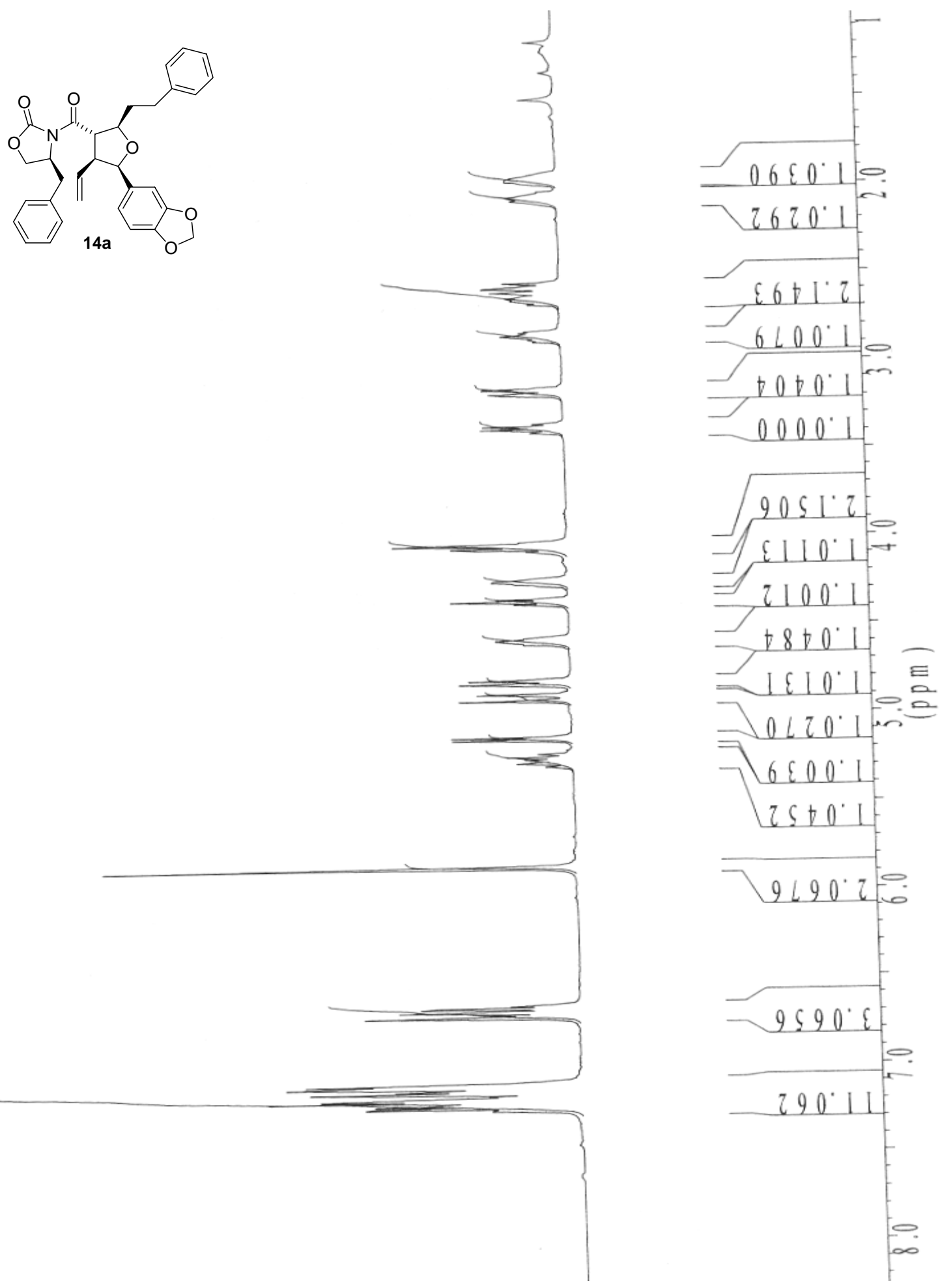
Compound 14a (Table 2, Entry 4)
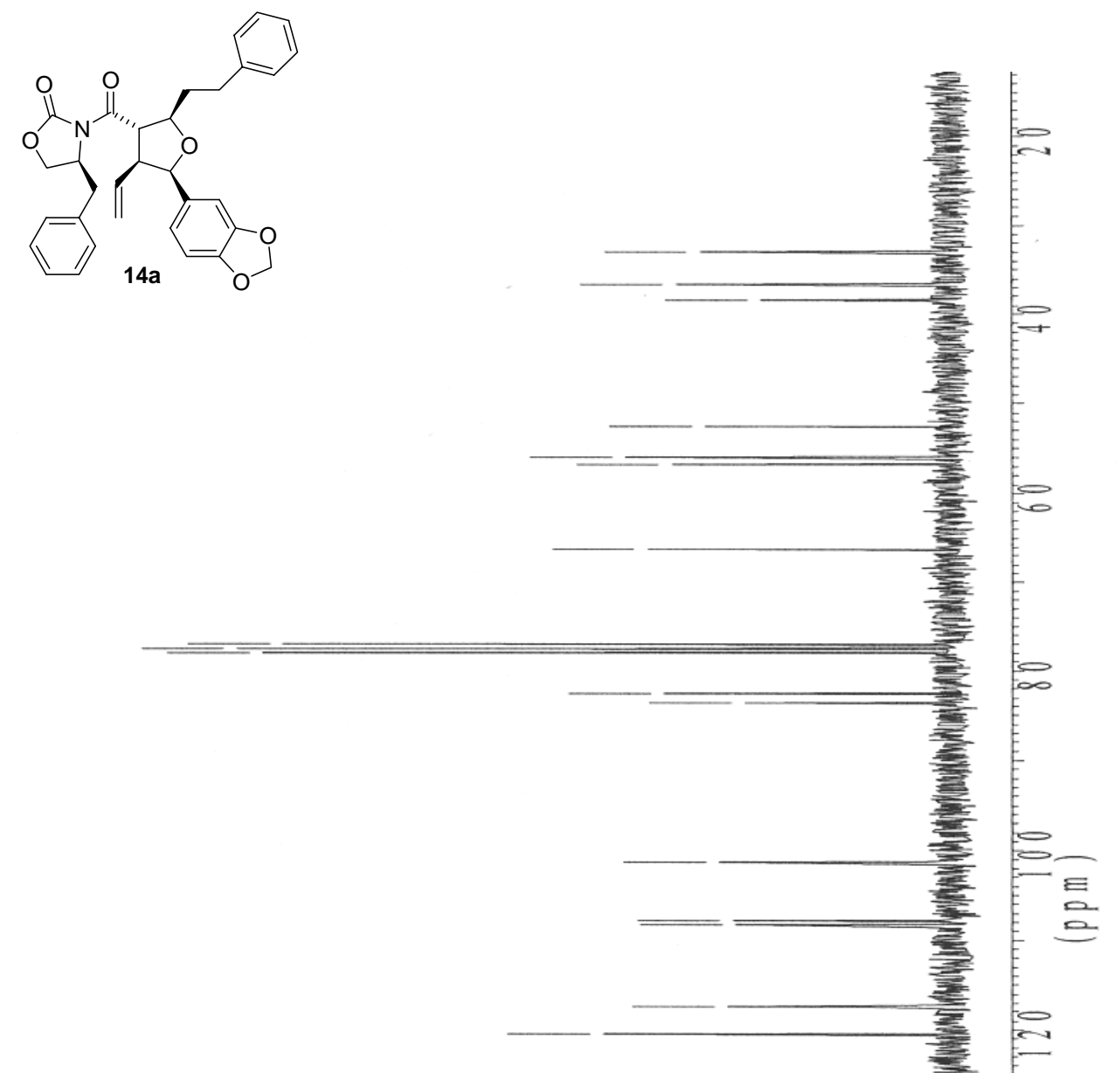
Compounds 15a/15b + 15c (unknown stereochemistry) (11:78:11, Table 1, Entry 7)
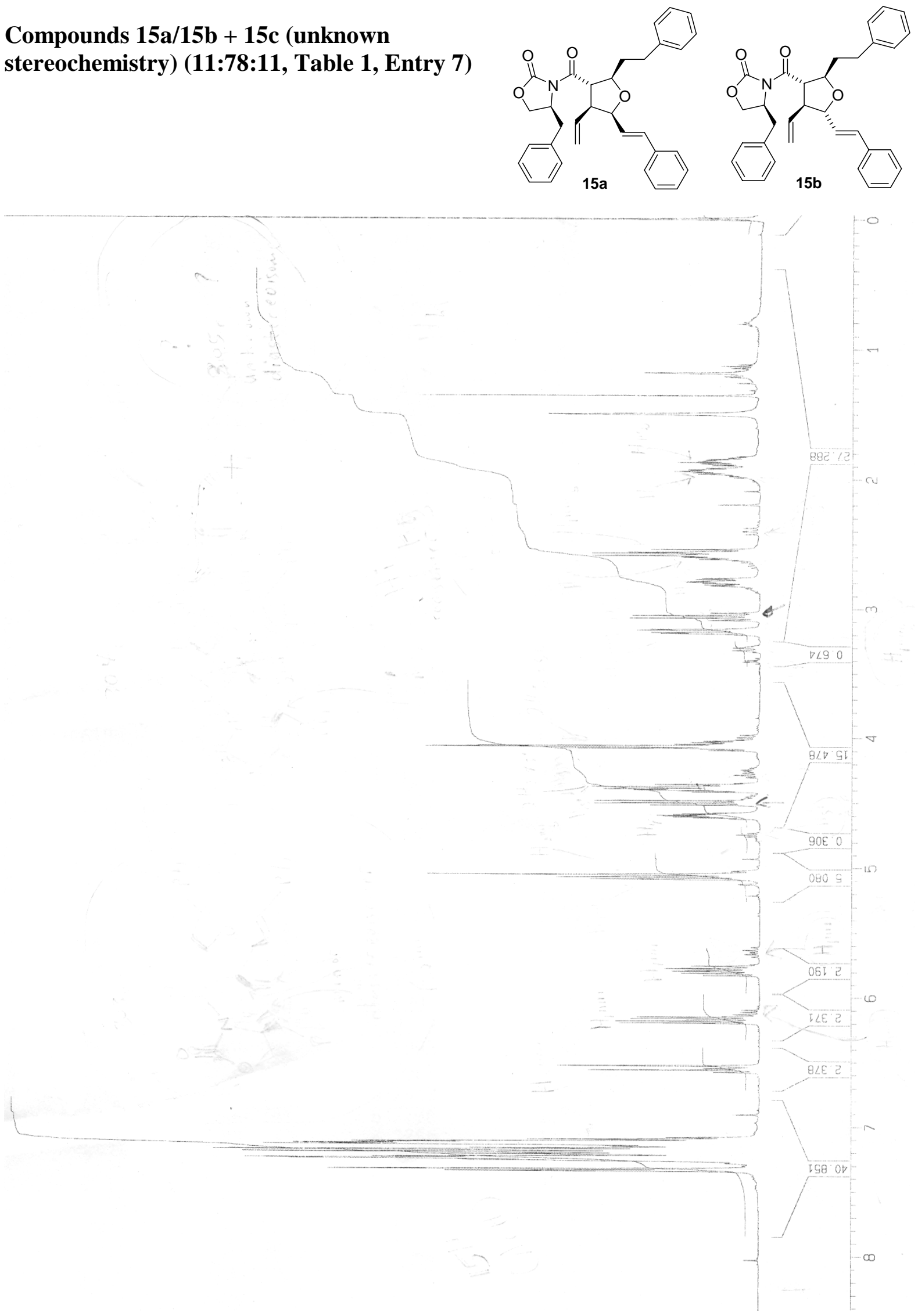
Compounds 15a/15b $+15 c$ (unknown

stereochemistry) (11:78:11, Table 1, Entry 7)
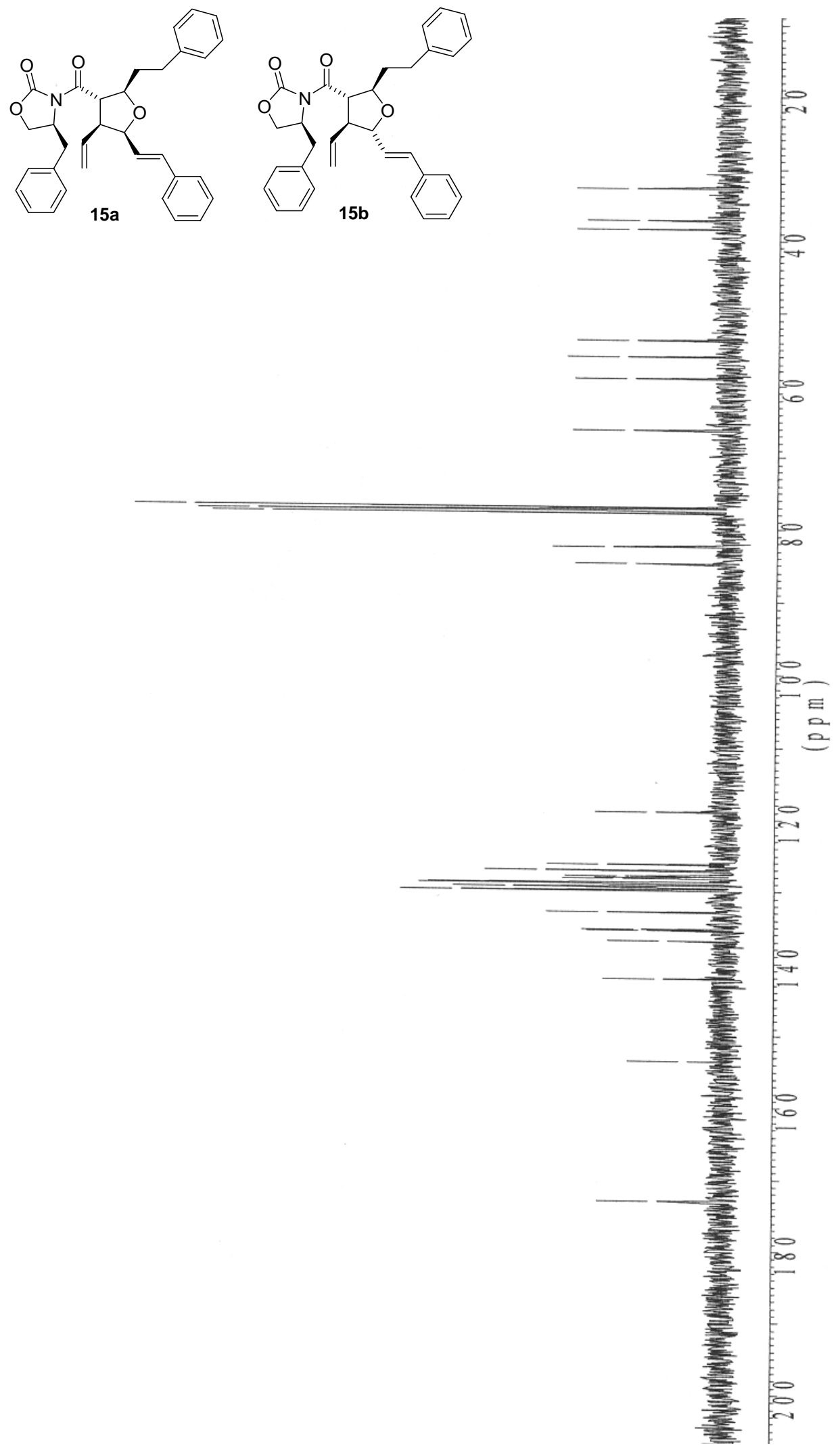


\section{Compounds 15a/15b (97:3, Table 2, Entry 5)}
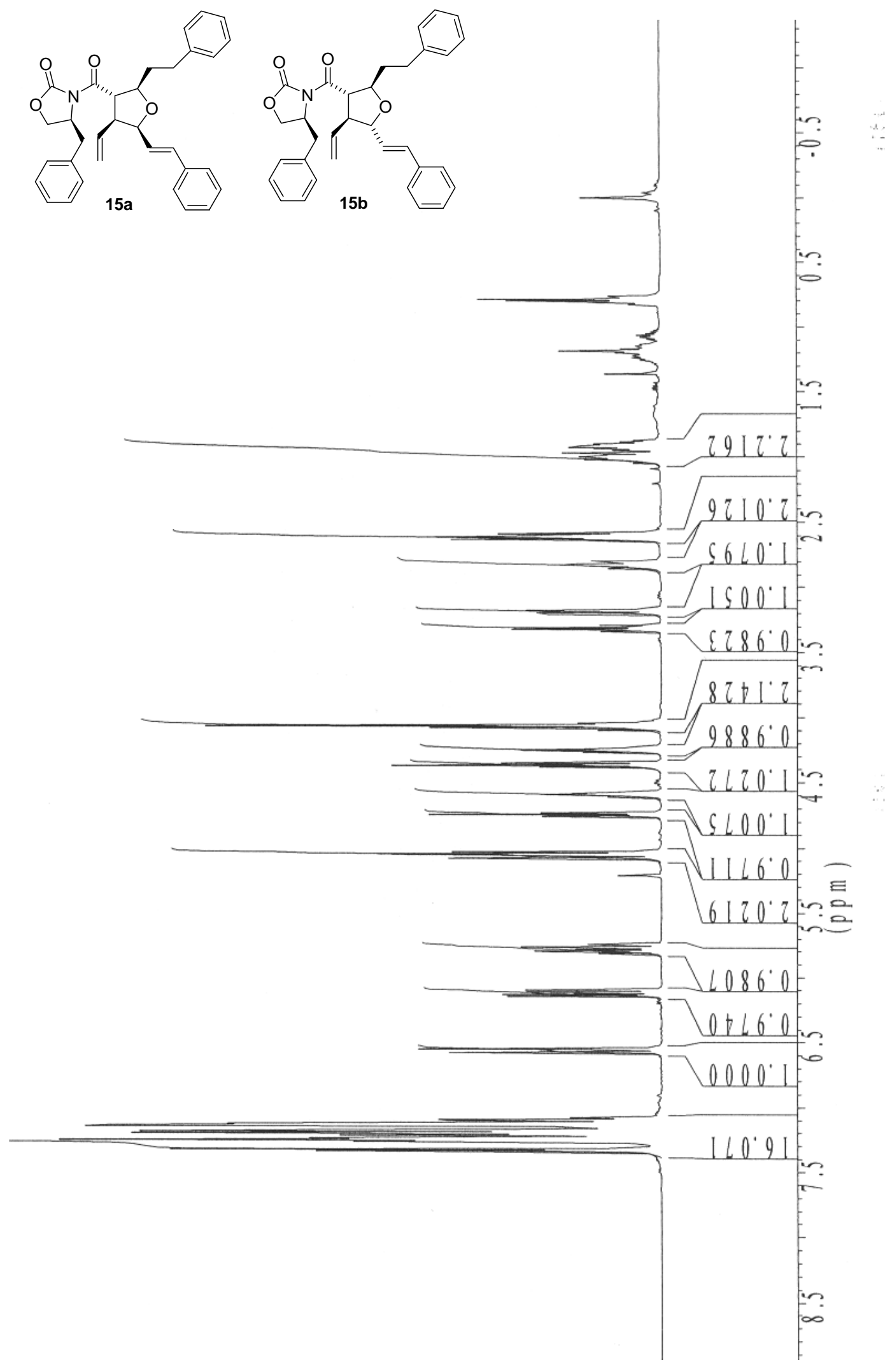
Compounds 15a/15b (97:3, Table 2, Entry 5)

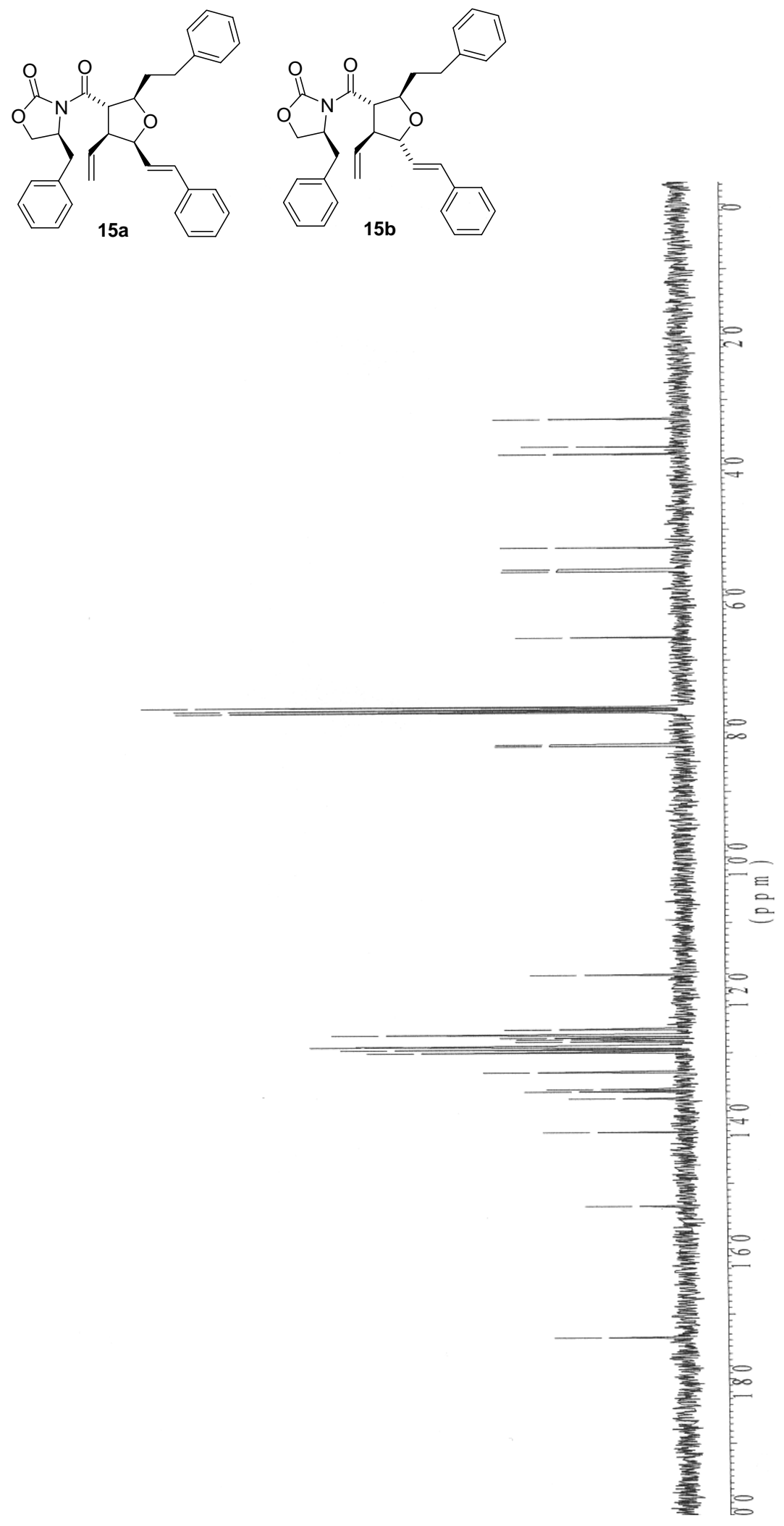

34 
Compound 16 a (Table 2, Entry 6)
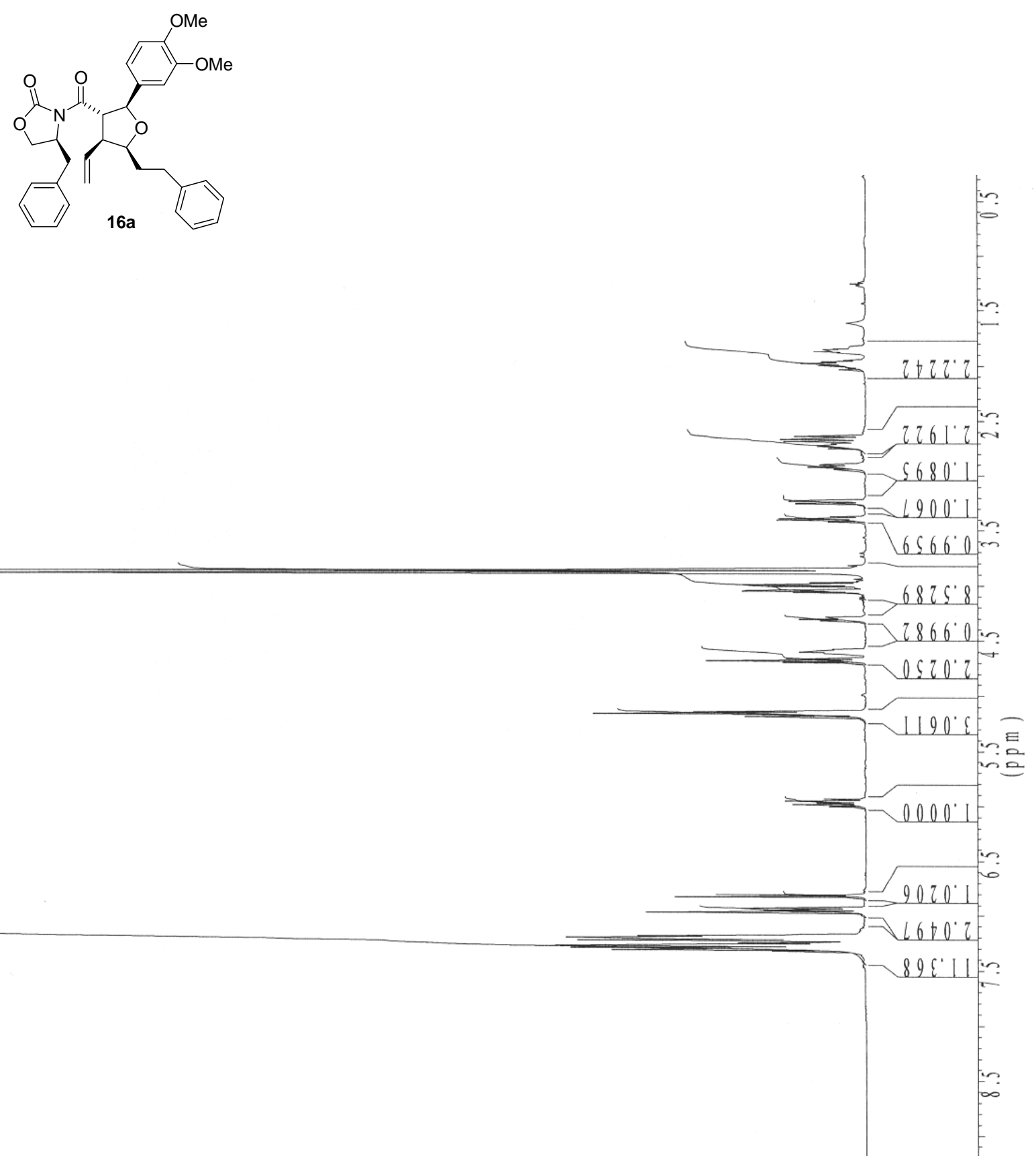
Compound 16 a (Table 2, Entry 6)
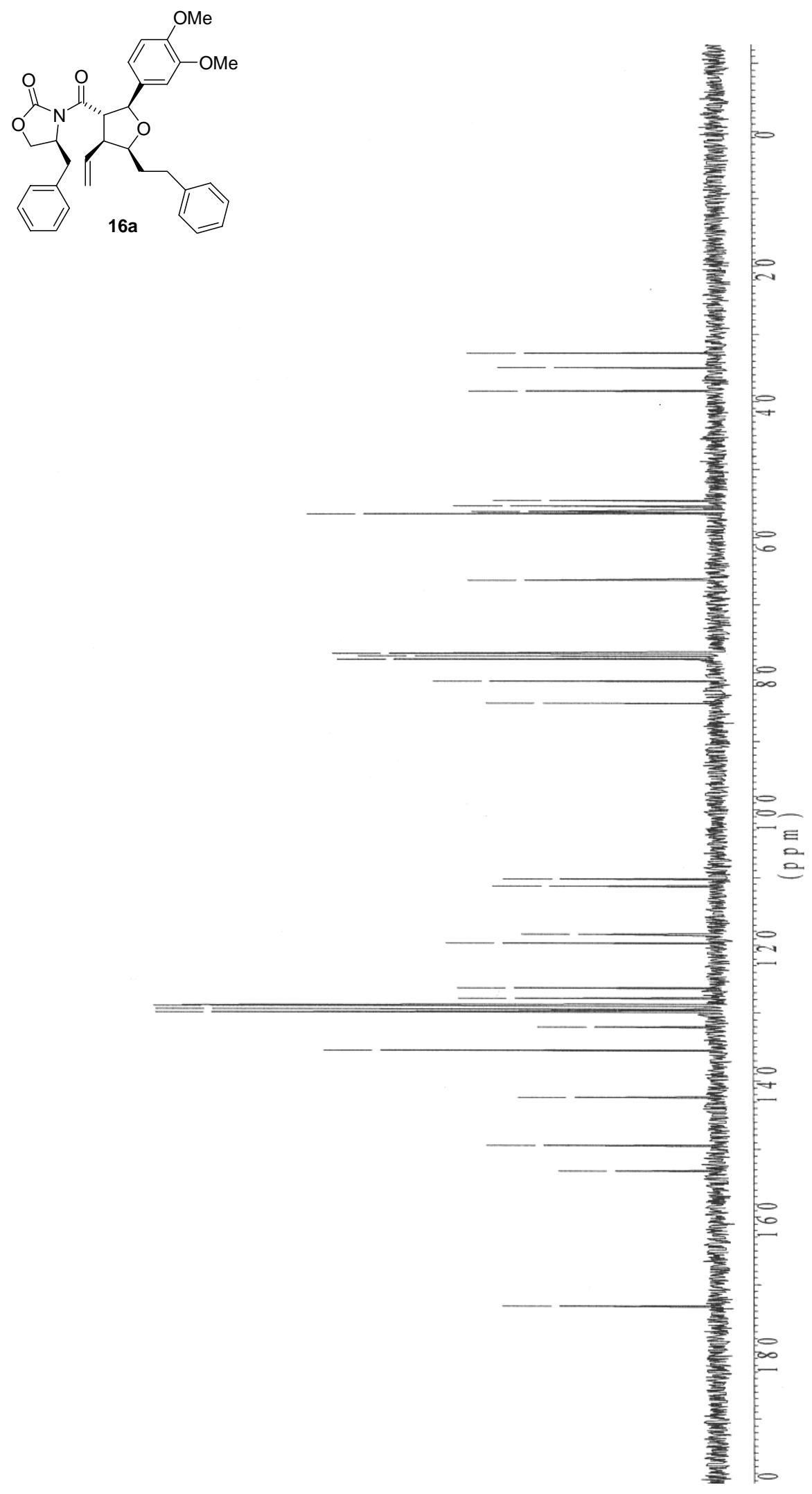


\section{Compound 17a (Table 2, Entry 7)}
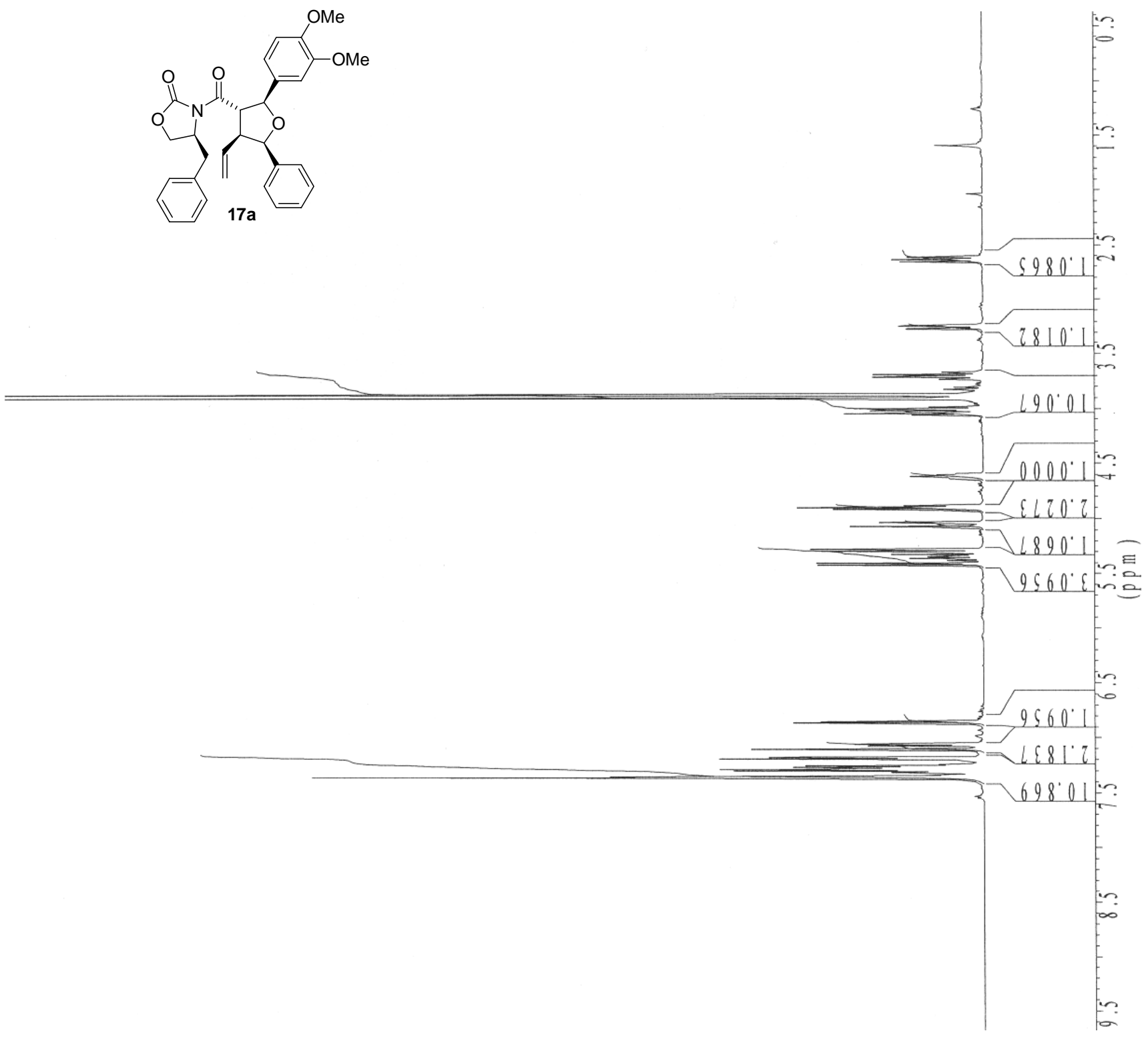


\section{Compound 17a (Table 2, Entry 7)}
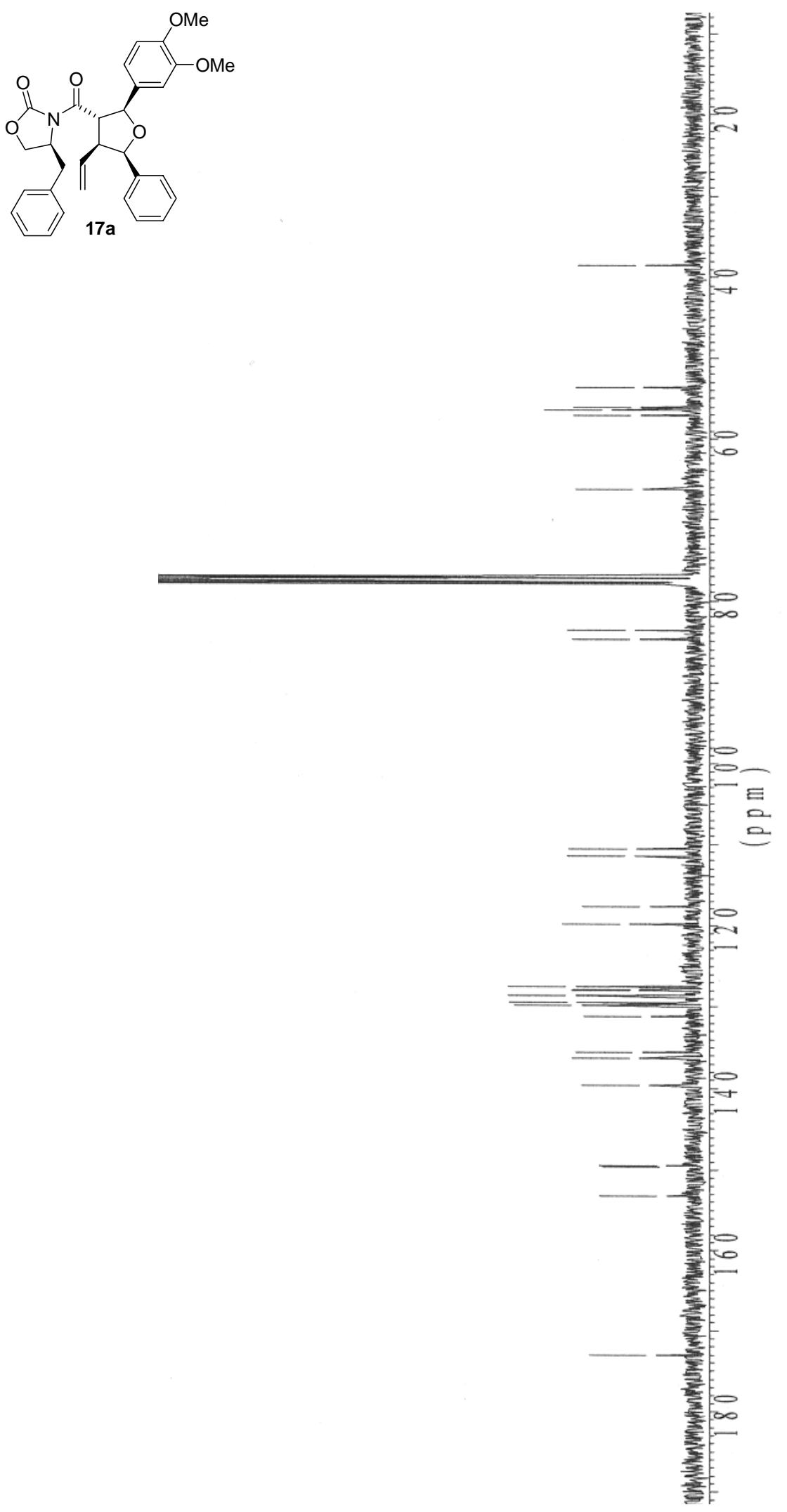


\section{Compounds 18a/18b (91:9, Table 2, Entry 8)}

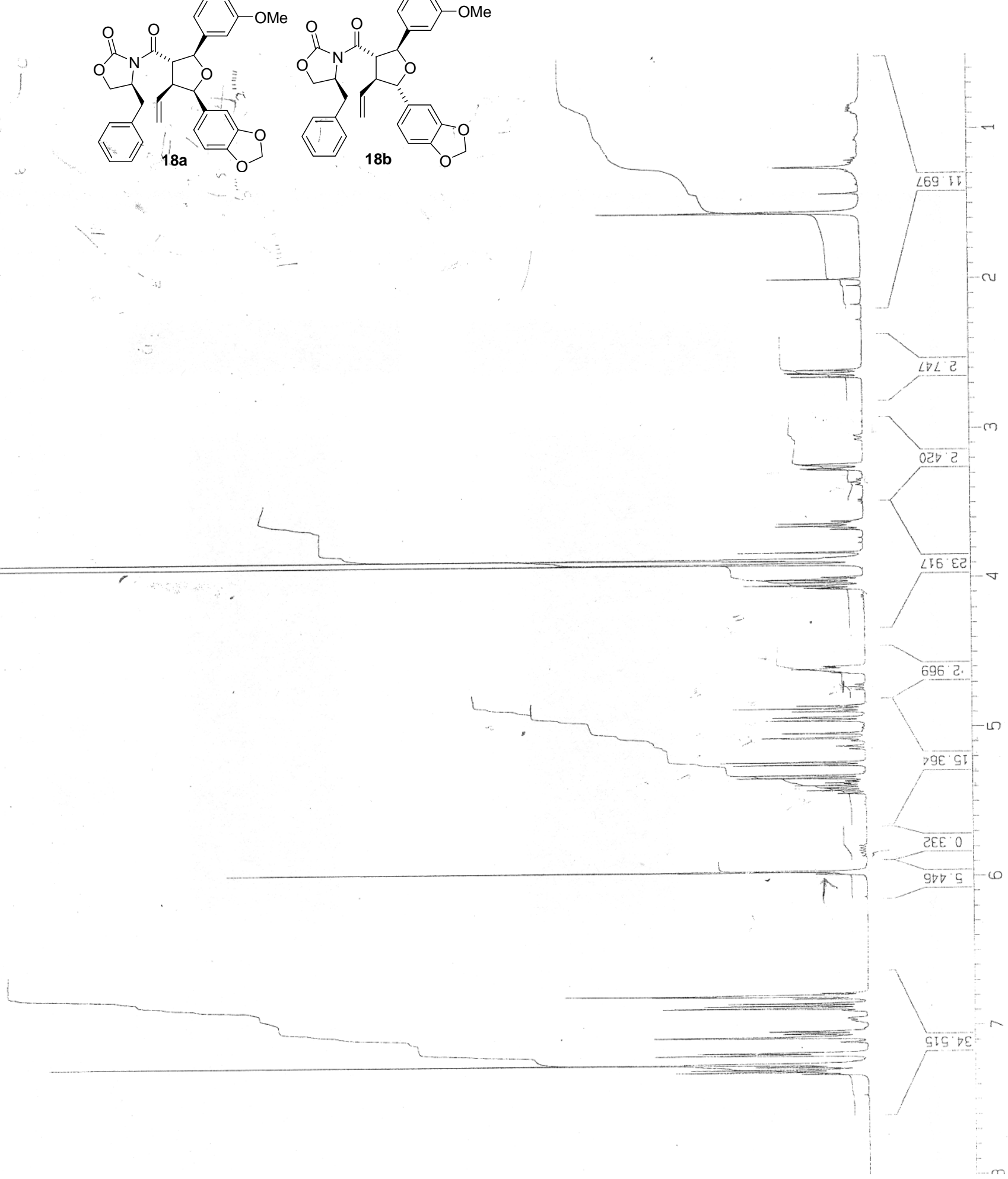




\section{Compounds 18a/18b (91:9, Table 2, Entry 8)}
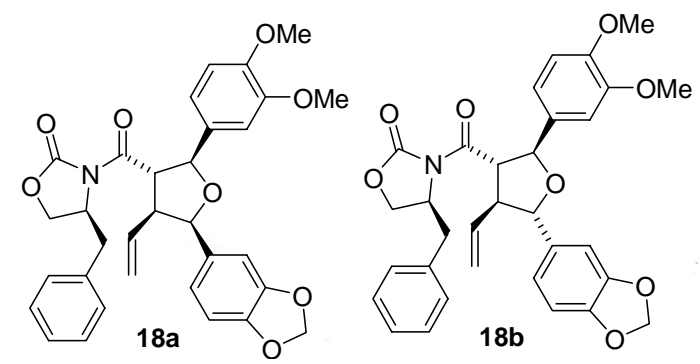

Me

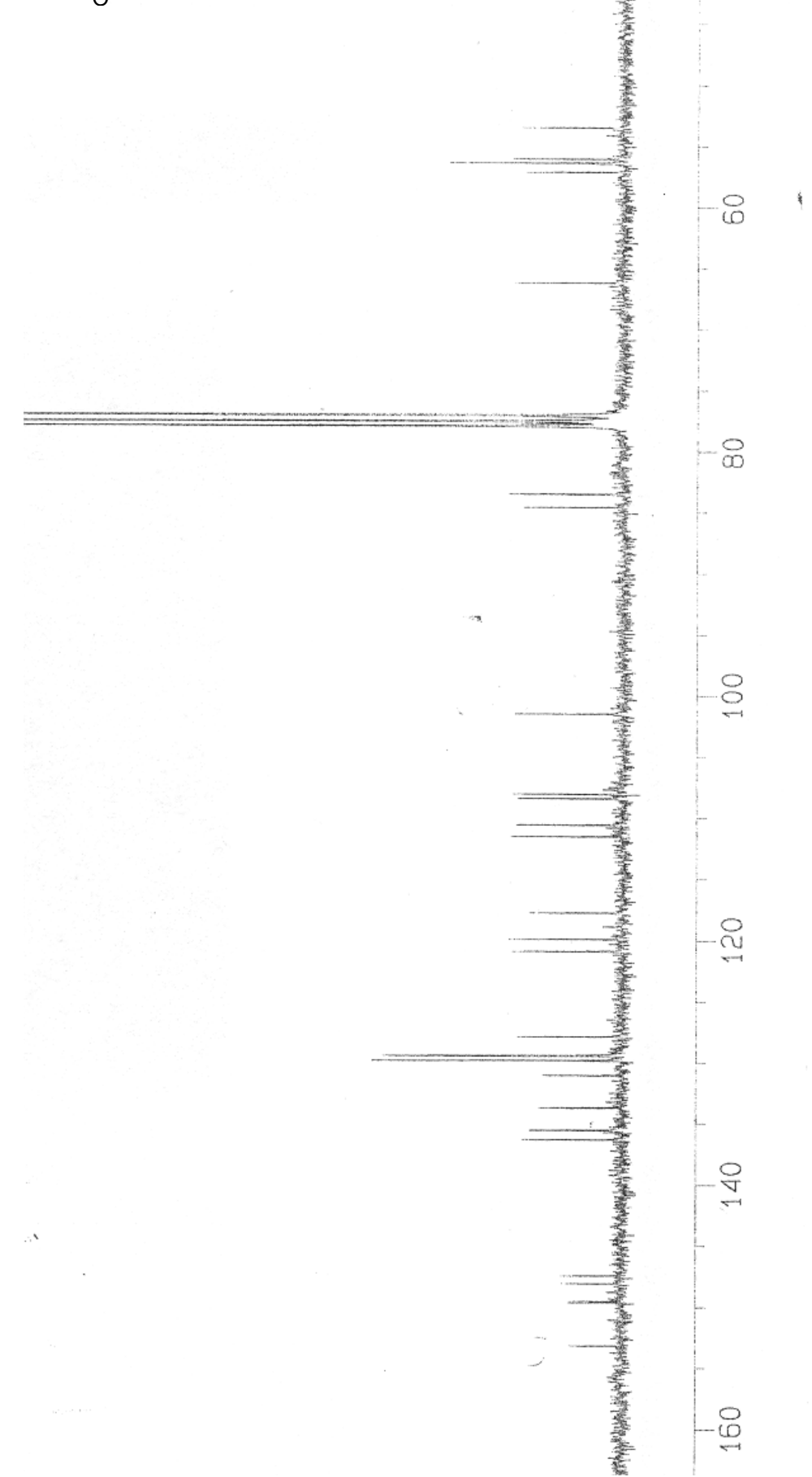




\section{Compound 19}

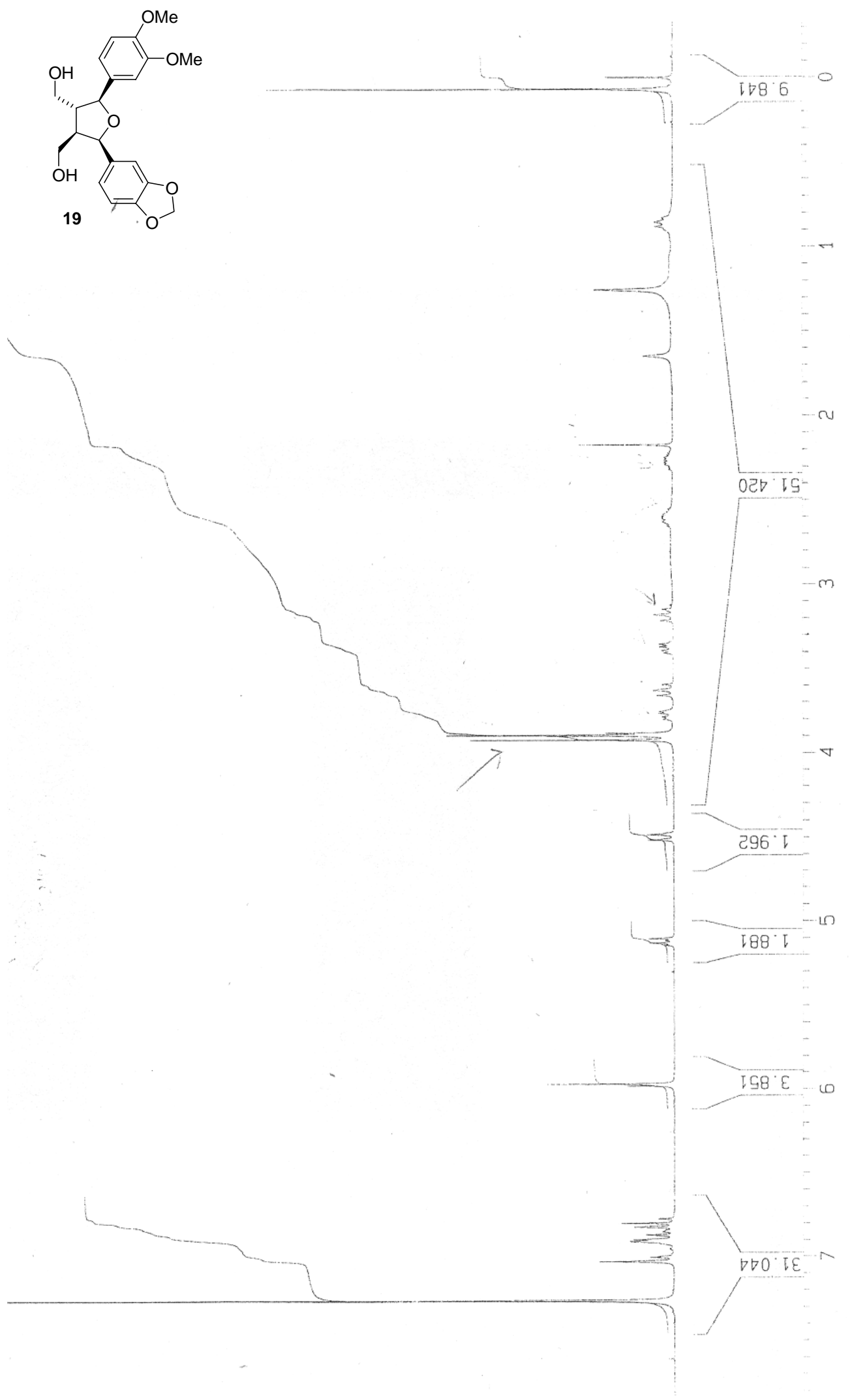




\section{Compound 19}
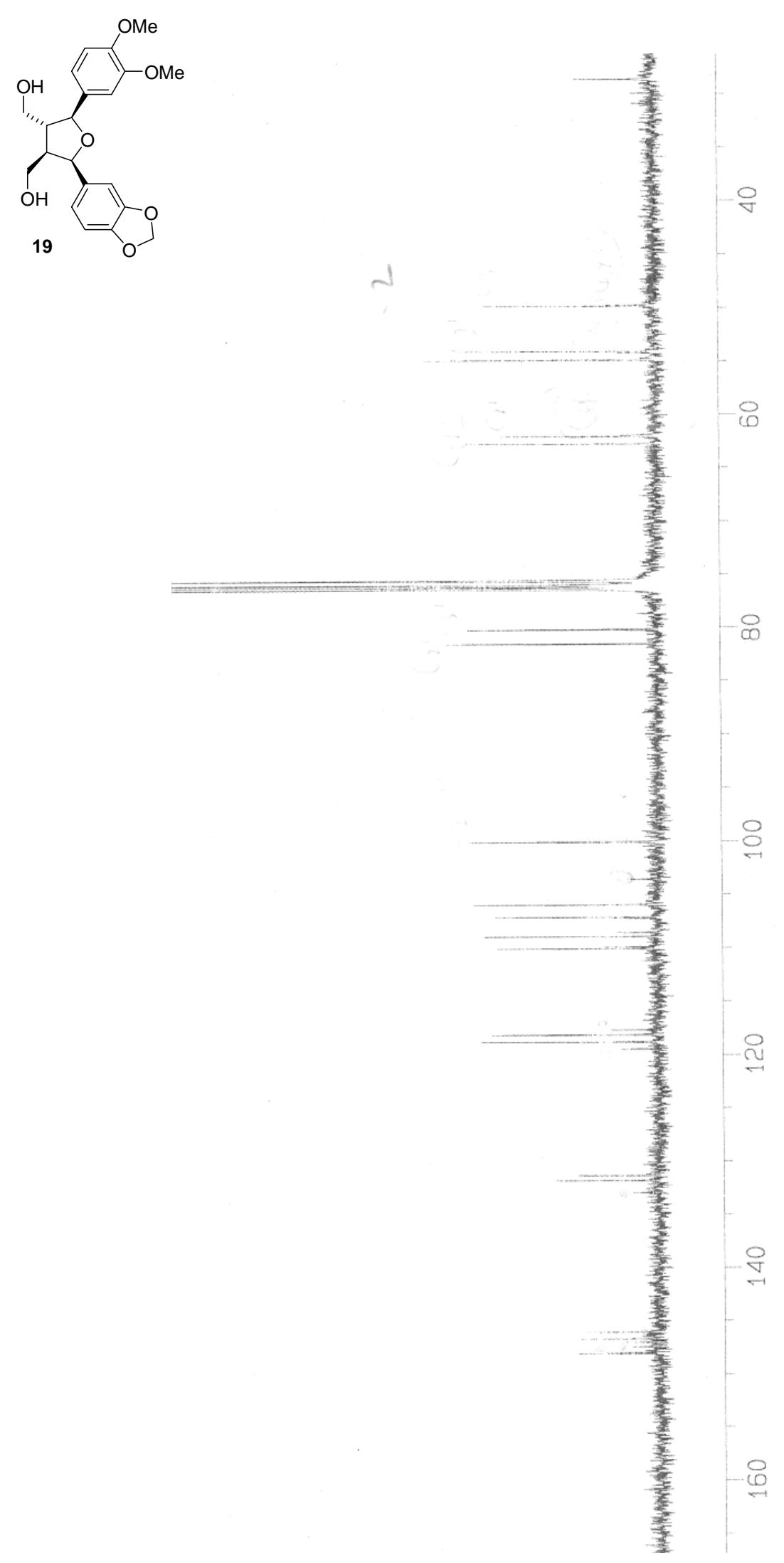
Compound 1 (virgatusin)
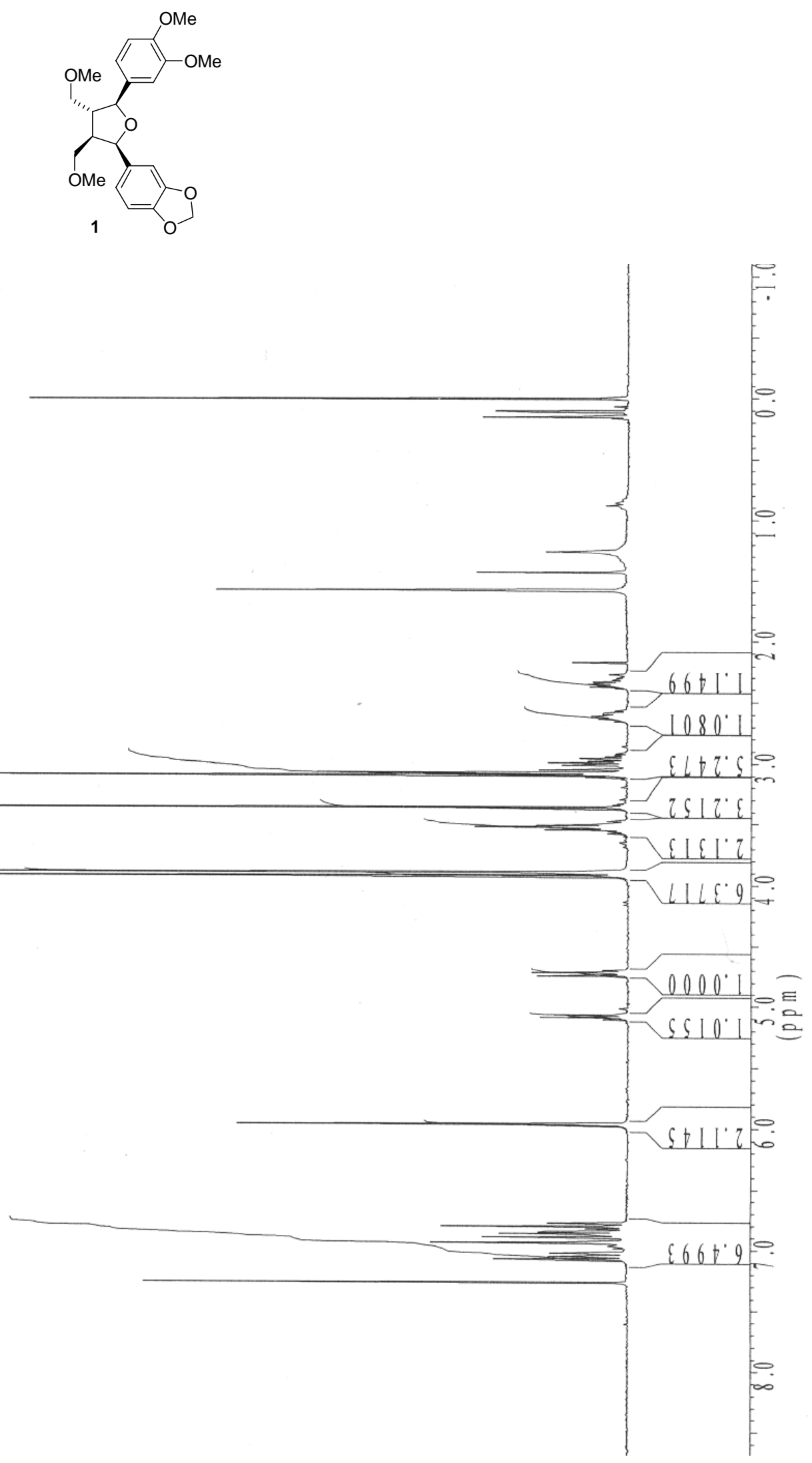
Compound 1 (virgatusin)
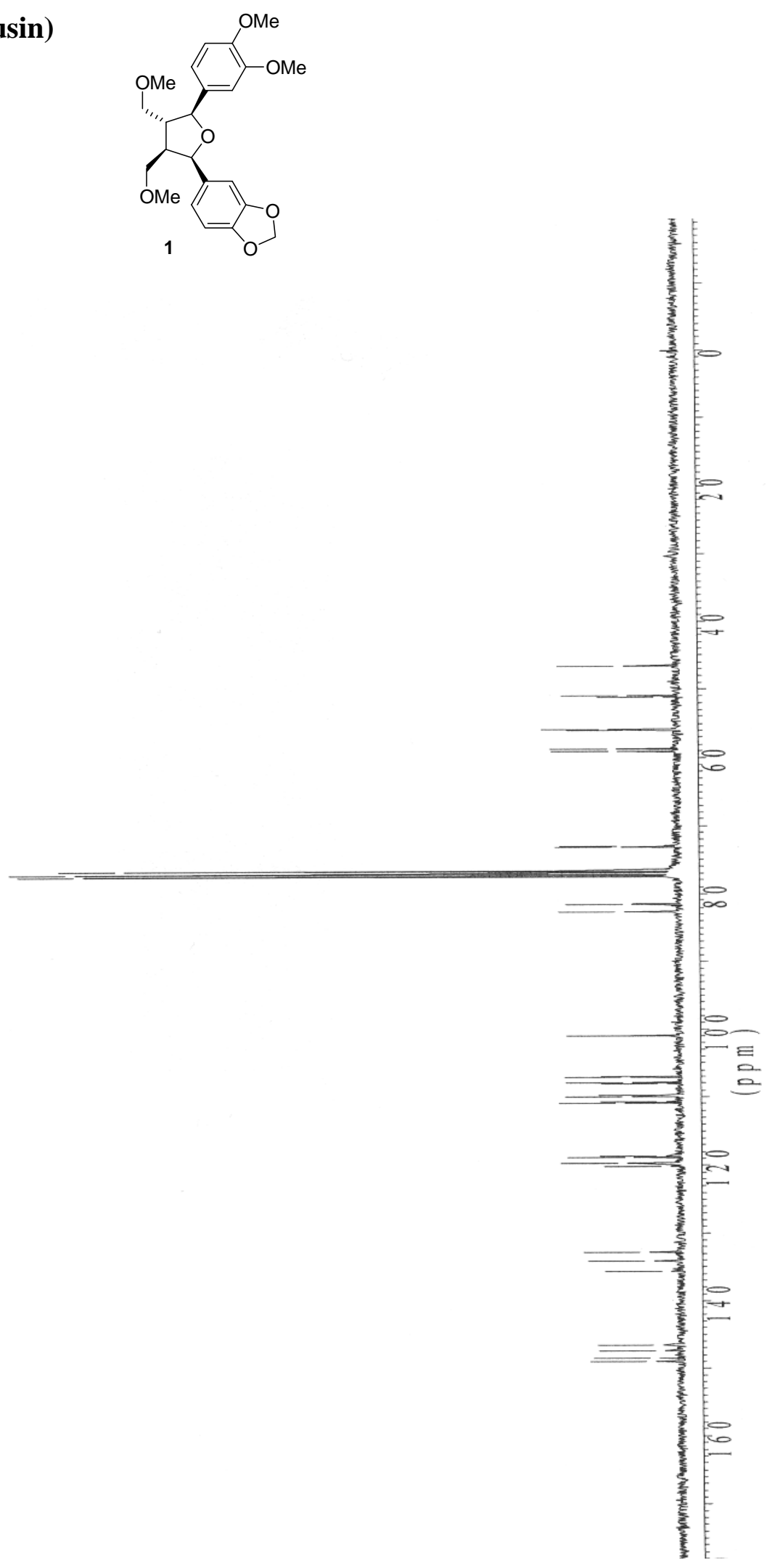